\title{
Modifying tetramethyl-nitrophenyl-imidazoline with amino acids: design, synthesis, and 3D-QSAR for improving inflammatory pain therapy
}

This article was published in the following Dove Press journal:

Drug Design, Development and Therapy

22 April 2015

Number of times this article has been viewed

\author{
Xueyun Jiang' \\ Yuji Wang' \\ Haimei Zhu' \\ Yaonan Wang' \\ Ming Zhao ${ }^{1,2}$ \\ Shurui Zhao' \\ Jianhui $\mathrm{Wu}^{\prime}$ \\ Shan $\mathrm{Li}^{\prime}$ \\ Shiqi Peng'
}

'Beijing Area Major Laboratory of Peptide and Small Molecular Drugs, Engineering Research Center of Endogenous Prophylactic of Ministry of Education of China, Beijing Laboratory of Biomedical Materials, College of Pharmaceutical Sciences, Capital Medical University, Beijing, People's Republic of China; ${ }^{2}$ Faculty of Biomedical Science and Environmental Biology, Kaohsiung Medical University, Kaohsiung, Taiwan

Correspondence: Shiqi Peng/Ming Zhao College of Pharmaceutical Sciences, Capital University of Medical Sciences, No. 10 Xitoutiao, You An Men, Beijing 100069, People's Republic of China Tel +86 I0 839| I528

Fax: +86 I0 82802482

Email shiqipeng@।63.com/sqpeng@ bjmu.edu.cn; mingzhao@bjmu.edu.cn

\begin{abstract}
With the help of pharmacophore analysis and docking investigation, 15 novel 1-(4,4,5,5-tetramethyl-2-(3-nitrophenyl)-4,5-dihydroimidazol-1-yl)-oxyacetyl-L-amino acids $(6 \mathrm{a}-\mathrm{o})$ were designed, synthesized, and assayed. On tail-flick and xylene-induced ear edema models, $10 \mu \mathrm{mol} / \mathrm{kg}$ 6a-o exhibited excellent oral anti-inflammation and analgesic activity. The dose-dependent assay of their representative $6 \mathrm{f}$ indicates that the effective dose should be $3.3 \mu \mathrm{mol} / \mathrm{kg}$. The correlation of the three-dimensional quantitative structure-activity relationship with the docking analysis provides a basis for the rational design of drugs to treat inflammatory pain.
\end{abstract}

Keywords: tetramethylimidazoline, analgesic, anti-inflammatory, 3D-QSAR

\section{Introduction}

A variety of etiologies such as inflammation and neural destruction can complicate chronic pain. Though neuropathic pain is simply accompanied by hypersensitivity to mechanical or thermal stimulus, ${ }^{1-4}$ inflammatory pain is accompanied by various painful responses to injury of peripheral tissue, trauma, infection, surgery, burns, or related diseases..$^{5-8}$ Treating inflammatory pain represents a medical need, and a lot of agents such as pyridazin derivatives, ${ }^{9}$ pyrazine $\mathrm{N}$-acylhydrazone derivatives,${ }^{10}$ oxadiazole derivatives, ${ }^{11}$ pyrazolone-pyridazine conjugates, ${ }^{12}$ and triazine derivatives ${ }^{13}$ were reported to possess anti-inflammation and analgesic activities. Besides, 1-hydroxyland 1-oxyl-tetramethylimidazolines were found to be analgesic pharmacophores, of which 1-oxyl-2-(3'-nitrophen-1'-yl)-4,4,5,5-tetramethylimidazoline and 1-hydroxyl-2(3'-nitrophen-1'-yl)-4,4,5,5-tetramethylimidazoline had the highest efficacy; ie, if the 2-position of 4,4,5,5-tetramethylimidazoline was 3-nitro-phenyl, the pharmacophore will possess the highest analgesic activity. ${ }^{14,15}$ The merge of the characteristics of the structures in the blue box and 2-(3'-nitrophen-1'-yl)-4,4,5,5-tetramethylimidazoline had the highest analgesic activity this paper designed 1-(4,4,5,5-tetramethyl-2-(3-nitrophenyl)-4,5dihydroimidazol-1-yl)-oxyacetyl-L-amino acids (TMNPIZA) as the therapeutic agents for inflammatory pain, docked them into the active site of cyclooxygenase-2 (COX-2) enzyme, found them properly fiting the active site, and observed the important interactions of TMNPIZA with Ser530, Phe518, Arg513, and Ser353 in particular (Figure 1).

In this context, here we present the preparation and evaluation of the oral analgesic and anti-inflammatory activities of 15 novel TMNPIZA on tail-flick and xylene-induced ear edema mouse models, respectively, as well as analyze their structure-activity relationship with Discovery Studio 4.0 three-dimensional quantitative structure-activity relationship (3D-QSAR) module. 

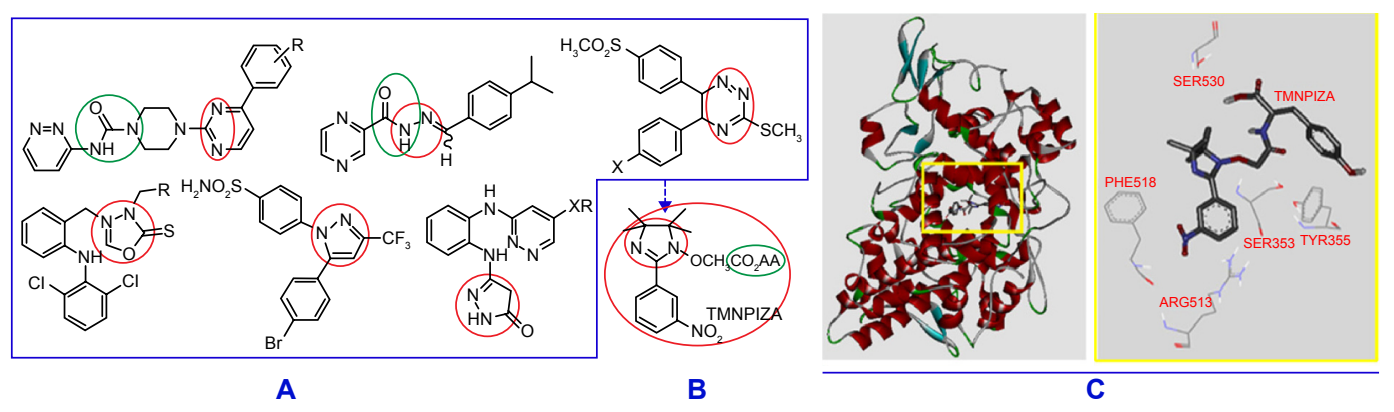

Figure I Design diagram: (A) the merge of the known structures of clinical anti-inflammatory pain agents in the blue box and tetramethylimidazolines been analgesic pharmacophore resulting in novel structure of TMNPIZA; (B) TMNPIZA fitting the active site of COX-2 enzyme and having a desirable docking feature; (C) estimating TMNPIZA being able to treat inflammatory pain, the feature of TMNPIZA in the active site of COX-2 is highlighted with a yellow box and interactions of TMNPIZA with the amino acid residues of the active site is clarified by amplifying the yellow box.

Abbreviations: AA, amino acid; TMNPIZA, I-(4,4,5,5-tetramethyl-2-(3-nitrophenyl)-4,5-dihydroimidazol-I-yl)-oxyacetyl-L-amino acids; COX-2, cyclooxygenase-2.

\section{Materials and methods Chemical synthesis \\ General method}

All the reactions were carried out under nitrogen (1 bar). ${ }^{1} \mathrm{H}(300$ and $500 \mathrm{MHz})$ and ${ }^{13} \mathrm{C}(75$ and $125 \mathrm{MHz})$ nuclear magnetic resonance (NMR) spectra were recorded on Bruker Avance II-300 and Avance II-500 spectrometers for solution DMSO- $d_{6}$ or $\mathrm{CDCl}_{3}$ with tetramethylsilane as internal standard. Infrared (IR) spectra were recorded with a Perkin-Elmer 983 instrument. Electrospray ionization mass spectrometry (ESI/MS) was determined on Waters Micromass Quattro Micro ZQ2000. Melting points were measured on an XT5 hot stage microscope (Beijing key electro-optic factory). All L-amino acids were purchased from China Biochemical Corp. Thin-layer chromatography (TLC) was made with Qingdao silica gel $\mathrm{GF}_{254}$. Chromatography was performed with Qingdao silica gel $\mathrm{H}_{60}$ or Sephadex- $\mathrm{LH}_{20}$. The purities of the intermediates were measured on TLC (Merck silica gel plates of type $60 \mathrm{~F}_{254}, 0.25 \mathrm{~mm}$ layer thickness, three systems of mixed solvents) and showed a single component, while the purities of the products were measured on high-performance liquid chromatography (Waters, $\mathrm{C}_{18}$ column $4.6 \times 150 \mathrm{~mm}$, three elution systems of mixed solvents) and more than $96 \%$. All solvents were distilled and dried before use according to literature procedures. Optical rotations were determined with a Jasco P-1020 Polarimeter at $20^{\circ} \mathrm{C}$. The statistical analysis of all the biological data was carried out by use of analysis of variance (ANOVA) test. $P<0.05$ is considered significant.

\section{Synthesis}

\section{2,3-dimethyl-2,3-dinitrobutane}

At $-5^{\circ} \mathrm{C}$, to a solution of $34.5 \mathrm{~g}(0.39 \mathrm{~mol})$ of 2-nitropropane in $65 \mathrm{~mL}$ of aqueous $\mathrm{NaOH}(6.0 \mathrm{M}) 10 \mathrm{~mL}(0.19 \mathrm{~mol})$ of $\mathrm{Br}_{2}$ was added dropwise within 1 hour. With stirring, $128 \mathrm{~mL}$ of ethanol was added, and this solution was then stirred at $84^{\circ} \mathrm{C}$ for 3 hours. The hot reaction mixture was transferred into $400 \mathrm{~mL}$ of ice water. The formed colorless crystals were collected by filtration to yield $25 \mathrm{~g}$ (73\%) of the title compound. $\mathrm{Mp} 110^{\circ} \mathrm{C}-112^{\circ} \mathrm{C}$.

\section{2,3-dihydroxylamino-2,3-dimethylbutane}

To a solution of $17.6 \mathrm{~g}$ of 2,3-dimethyl-2,3-dinitrobutane $(0.1 \mathrm{~mol})$ in $300 \mathrm{~mL}$ of tetrahydrofuran (THF) and $50 \mathrm{~mL}$ of water of $9^{\circ} \mathrm{C} 27 \mathrm{~g}$ of $\mathrm{Zn}$ powder was added to get a slurry-like mixture. To this stirring mixture a solution of $43 \mathrm{~g}$ of $\mathrm{NH}_{4} \mathrm{Cl}$ $(0.8 \mathrm{~mol})$ in $150 \mathrm{~mL}$ of $\mathrm{H}_{2} \mathrm{O}$ was added dropwise within 2 hours and was then stirred at $10^{\circ} \mathrm{C}$ for 1 hour and stored at $4^{\circ} \mathrm{C}$ for 16 hours. The slurry-like mixture was filtered, the collected precipitates were washed with THF $(100 \mathrm{~mL} \times 4)$, and diethyl ether $(50 \mathrm{~mL} \times 3)$ was added to provide $59 \mathrm{~g}$ of precipitates. The solution was evaporated under vacuum to remove THF. Then, the solution was protected from air, to which $50 \mathrm{~g}$ of sodium carbonate and $30 \mathrm{~g}$ of sodium chloride were added with cooling. The solids were continuously extracted with $400 \mathrm{~mL}$ of chloroform over 18 hours to provide $9.4 \mathrm{~g}(63 \%)$ of the title compound as a colorless powder. Mp $182^{\circ} \mathrm{C}$.

\section{I,3-dihydroxyl-2-(3-nitrophen-I-yl)-4,4,5,5- tetramethylimidazolidine (I)}

A solution of $296 \mathrm{mg}$ ( $2 \mathrm{mmol}$ ) of 2,3-bis(hydroxylamino)2,3-dimethylbutane and $302 \mathrm{mg}$ ( $2 \mathrm{mmol}$ ) of 3-nitrobenzaldehyde in $3 \mathrm{~mL}$ of methanol was stirred at room temperature for 16 hours. The title compound was collected by filtration to provide $421 \mathrm{mg}$ (75\%) of the title compound. $\mathrm{Mp} 179^{\circ} \mathrm{C}-81^{\circ} \mathrm{C}$. ESI-MS $(\mathrm{m} / \mathrm{z}) 281[\mathrm{M}+\mathrm{H}]^{+}$. IR $(\mathrm{KBr})$ $3,315,1,530,1,360,1,600,875,790,685 \mathrm{~cm}^{-1} .{ }^{1} \mathrm{H}$ NMR (300 MHz, DMSO-d6) $\delta / \mathrm{ppm}=8.03$ (s, 2H), 7.77 (s, 1H), 7.57 (d, $J=7.5 \mathrm{~Hz}, 1 \mathrm{H}), 7.34$ (d, $J=6.0 \mathrm{~Hz}, 1 \mathrm{H}), 7.04$ (dd, $J=8.7 \mathrm{~Hz}, J=8.1 \mathrm{~Hz}, 1 \mathrm{H}), 4.19$ (s, 1H), 0.52 (s, 12H). 


\section{I-hydroxyl-2-(3-nitrophen- I-yl)-4,4,5,5-}

tetramethylimidazoline (2)

A suspension of $281 \mathrm{mg}(1 \mathrm{mmol})$ of (1) and $360 \mathrm{mg}$ of anhydrous $\mathrm{MgSO}_{4}$ in $40 \mathrm{~mL}$ of anhydrous THF was stirred at $80^{\circ} \mathrm{C}$ for 24 hours, and $\mathrm{TLC}\left(\mathrm{CHCl}_{3} / \mathrm{CH}_{3} \mathrm{OH}, 20: 1\right)$ indicated the complete disappearance of (1). The reaction mixture was cooled to room temperature. After removal of $\mathrm{MgSO}_{4}$ by filtration, the filtrate was evaporated to dryness. The residue was purified on chromatography column of silica gel $\left(\mathrm{CHCl}_{3} /\right.$ $\mathrm{CH}_{3} \mathrm{OH}, 30: 1$ ) to give $174 \mathrm{mg}$ (66\%) of the title compound as yellow powder. Mp $97^{\circ} \mathrm{C}-99^{\circ} \mathrm{C}$. EI-MS $(\mathrm{m} / \mathrm{z}) 263[\mathrm{M}]^{+}$. IR (KBr) 3,345, 1,602, 1,513, 1,455, 1,370, 811, 790, $680 \mathrm{~cm}^{-1}$. ${ }^{1} \mathrm{H}$ NMR (300 MHz, $\left.\mathrm{CDCl}_{3}-\mathrm{d}\right) \delta / \mathrm{ppm}=8.55$ (s, 1H), 8.22 $(\mathrm{d}, J=7.20 \mathrm{~Hz}, 1 \mathrm{H}), 7.99$ (d, $J=7.21 \mathrm{~Hz}, 1 \mathrm{H}), 7.55(\mathrm{t}, J=7.21 \mathrm{~Hz}$, 1H), 2.65 (s, 1H), $1.15(\mathrm{~s}, 6 \mathrm{H}), 1.13(\mathrm{~s}, 6 \mathrm{H})$.

\section{Ethyl 2-(4,4,5,5-tetramethyl-2-(3-nitrophenyl)-4,5-} dihydroimidazol-I-ylo-xy)acetate (3)

At $0^{\circ} \mathrm{C}$, to a solution of $500 \mathrm{mg}(1.77 \mathrm{mmol})$ of (2) in $40 \mathrm{~mL}$ of THF and $10 \mathrm{~mL}$ of $80 \mathrm{mg} 2.12 \mathrm{mmol}$ of $\mathrm{NaH}$ was added and stirred for 30 minutes. To this suspension, $0.3 \mathrm{~mL}(2.12 \mathrm{mmol})$ of $\mathrm{BrCH}_{2} \mathrm{COOC}_{2} \mathrm{H}_{5}$ was added and stirred at room temperature for 5 hours, and $\mathrm{TLC}\left(\mathrm{CHCl}_{3}\right.$ : $\left.\mathrm{CH}_{3} \mathrm{OH}=30: 1\right)$ indicated the complete disappearance of (2). The reaction mixture was filtered, the filtrate was evaporated under vacuum to remove THF, and the residue was dissolved in $200 \mathrm{~mL}$ of ethyl acetate. This solution was washed with saturated aqueous $\mathrm{NaHCO}_{3}(50 \mathrm{~mL} \times 3)$ and saturated aqueous $\mathrm{NaCl}(50 \mathrm{~mL} \times 2)$. The ethyl acetate phase was dried with anhydrous $\mathrm{Na}_{2} \mathrm{SO}_{4}$ over light and filtered, the filtrate was evaporated under vacuum, and the residue was purified with column chromatography (EtOAC:petroleum=1:3) to provide $529 \mathrm{mg}(82 \%)$ of the title compound as yellow syrup. ESI/ MS (m/z) $350[\mathrm{M}+\mathrm{H}]^{+} .{ }^{1} \mathrm{H}$ NMR (300 MHz, DMSO- $\left.d_{6}\right)$ : $\delta / \mathrm{ppm}=8.41(\mathrm{~s}, 1 \mathrm{H}), 8.34(\mathrm{~m}, 1 \mathrm{H}), 8.09$ (d, $J=7.8 \mathrm{~Hz}, 1 \mathrm{H})$, 7.74 (t, $J=7.8 \mathrm{~Hz}, 1 \mathrm{H}), 4.70$ (s, 2H), 4.11 (q, $J=7.2 \mathrm{~Hz}, 2 \mathrm{H})$, $1.22(\mathrm{~s}, 6 \mathrm{H}), 1.17$ (t, $J=7.2 \mathrm{~Hz}, 3 \mathrm{H}), 1.13(\mathrm{~s}, 6 \mathrm{H}) .{ }^{13} \mathrm{C} \mathrm{NMR}$ (75 MHz, DMSO- $\left.d_{6}\right)$ : $\mathrm{d}=168.66,162.45,147.85,134.79$, $132.80,130.43,125.21,123.15,74.08,73.02,68.40,61.00$, 23.88, 19.74, 14.41. Anal. Calcd for $\mathrm{C}_{17} \mathrm{H}_{23} \mathrm{~N}_{3} \mathrm{O}_{5}: \mathrm{C}, 58.44$; $\mathrm{H}, 6.64 ; \mathrm{N}, 12.03 ; \mathrm{O}, 22.90$.

\section{2-(4,4,5,5-tetramethyl-2-(3-nitrophenyl)-4,5-}

dihydroimidazol-I-yloxy)acetic acid (4)

At $0^{\circ} \mathrm{C}$, to a solution of $2.0 \mathrm{~g}(5.7 \mathrm{mmol})$ of (3) in $20 \mathrm{~mL}$ of methanol $4 \mathrm{~mL}$ of aqueous $\mathrm{NaOH}(4 \mathrm{M})$ was added. The reaction mixture was stirred at $0^{\circ} \mathrm{C}$ for 2 hours, and TLC (EtOAC: petroleum=1:3) indicated the complete disappearance of (3).
The reaction mixture was adjusted to $\mathrm{pH} 2$ and evaporated to remove methanol. The residue was extracted with ethyl acetate $(50 \mathrm{~mL} \times 3)$, the ethyl acetate phase was dried with anhydrous $\mathrm{Na}_{2} \mathrm{SO}_{4}$ over light and filtered, and the filtrate was evaporated under vacuum to provide $1.6 \mathrm{~g}(87 \%)$ of the title compound as yellow powder. $\mathrm{Mp} 66^{\circ} \mathrm{C}-69^{\circ} \mathrm{C}$; ESI/MS $(\mathrm{m} / \mathrm{z}) 320[\mathrm{M}-\mathrm{H}] .[\alpha]_{20}{ }^{\mathrm{D}=}=-7.5\left(\mathrm{c}=1.2, \mathrm{CH}_{3} \mathrm{OH}\right) .{ }^{1} \mathrm{H}$ NMR $\left(300 \mathrm{MHz}, \mathrm{DMSO}-d_{6}\right): \delta / \mathrm{ppm}=8.42(\mathrm{~s}, 1 \mathrm{H}), 8.32(\mathrm{~m}, 1 \mathrm{H})$, $8.10(\mathrm{~d}, J=7.8 \mathrm{~Hz}, 1 \mathrm{H}), 7.74(\mathrm{t}, J=7.8 \mathrm{~Hz}, 1 \mathrm{H}), 4.71(\mathrm{~s}, 2 \mathrm{H})$, $1.20(\mathrm{~s}, 6 \mathrm{H}), 1.12(\mathrm{~s}, 6 \mathrm{H}) \cdot{ }^{13} \mathrm{C}$ NMR $\left(75 \mathrm{MHz}, \mathrm{DMSO}-d_{6}\right)$ : $\mathrm{d}=169.14,163.10,148.52,134.95,132.63,131.25,124.87$, $122.87,76.12,66.40,58.75,23.60,20.20,14.97$. Anal. Calcd for $\mathrm{C}_{15} \mathrm{H}_{19} \mathrm{~N}_{3} \mathrm{O}_{5}: \mathrm{C}, 56.07 ; \mathrm{H}, 5.96 ; \mathrm{N}, 13.08$. Found C, 56.30; $\mathrm{H}, 6.15 ; \mathrm{N}, 13.30$.

General procedure of preparing I-(4,4,5,5-tetramethyl2-(3-nitrophenyl)-4,5-dihydroimidazol-I-yloxy)acetyl-Lamino acid benzylesters $(5 \mathrm{a}-0)$

At $0^{\circ} \mathrm{C}, 642 \mathrm{mg}(2.0 \mathrm{mmol})$ of (4), $270 \mathrm{mg}(2.0 \mathrm{mmol})$ of $\mathrm{HOBt}$, and $500 \mathrm{mg}$ ( $2.4 \mathrm{mmol})$ of Dicyclohexylcarbodiimide (DCC) was successively dissolved in $50 \mathrm{~mL}$ of anhydrous THF, and this solution was stirred at $0^{\circ} \mathrm{C}$ for 30 minutes. To this solution $2 \mathrm{mmol}$ of L-amino acid benzyleater was added and was adjusted to $\mathrm{pH} 8$. The reaction mixture was stirred at room temperature for 6 hours, and TLC indicated the complete disappearance of (4). The reaction mixture was filtered and the filtrate was evaporated under vacuum to remove THF. The residue was dissolved in $200 \mathrm{~mL}$ of ethyl acetate. This solution was washed with saturated aqueous $\mathrm{NaHCO}_{3}(50 \mathrm{~mL} \times 3)$ and saturated aqueous $\mathrm{NaCl}$ $(50 \mathrm{~mL} \times 2)$. The ethyl acetate phase was dried with anhydrous $\mathrm{Na}_{2} \mathrm{SO}_{4}$ over light and filtered, the filtrate was evaporated under vacuum, and the residue was purified with column chromatography (EtOAC:petroleum=1:1) to provide $5 \mathrm{a}-\mathrm{O}$.

\section{Benzyl (2S,3S)-3-methyl-2-(2-(4,4,5,5-tetramethyl-2-} (3-nitrophenyl)-4,5-dihydroimidazol-I-yloxy)acetamido) pentanoate $(5 \mathrm{a})$

Yield: $925 \mathrm{mg}$ (88\%). ESI/MS (m/z) $525[\mathrm{M}+\mathrm{H}]^{+} .{ }^{1} \mathrm{H}$ NMR $\left(300 \mathrm{MHz}, \mathrm{DMSO}-d_{6}\right): \delta / \mathrm{ppm}=8.43(\mathrm{t}, J=3.0 \mathrm{~Hz}, 1 \mathrm{H}), 8.33$ (m, 1H), $8.18(\mathrm{~d}, J=8.1 \mathrm{~Hz}, 1 \mathrm{H}), 8.12(\mathrm{~d}, J=8.1 \mathrm{~Hz}, 1 \mathrm{H})$, $7.71(\mathrm{t}, J=8.1 \mathrm{~Hz}, 1 \mathrm{H}), 7.35(\mathrm{~m}, 5 \mathrm{H}), 5.12(\mathrm{~m}, 2 \mathrm{H}), 4.53$ (s, 2H), $4.28(\mathrm{dd}, J=8.1 \mathrm{~Hz}, J=6.3 \mathrm{~Hz}, 1 \mathrm{H}), 1.78(\mathrm{~m}, 1 \mathrm{H})$, $1.32(\mathrm{~m}, 2 \mathrm{H}), 1.20(\mathrm{~s}, 6 \mathrm{H}), 1.12(\mathrm{~s}, 6 \mathrm{H}), 0.991(\mathrm{~m}, 6 \mathrm{H})$. ${ }^{13} \mathrm{C}$ NMR (75 MHz, DMSO- $\left.d_{6}\right): \delta / \mathrm{ppm}=171.49,167.78$, $162.49,147.97,136.21,134.75,132.87,130.42,128.87$, $128.60,128.55,125.20,123.12,75.42,72.91,68.34,66.47$, $56.49,36.79,25.10,23.90,15.78,11.48$. Anal. Calcd for 
$\mathrm{C}_{28} \mathrm{H}_{36} \mathrm{~N}_{4} \mathrm{O}_{6}: \mathrm{C}, 64.10 ; \mathrm{H}, 6.92 ; \mathrm{N}, 10.68$. Found: C, 64.29; $\mathrm{H}, 6.78 ; \mathrm{N}, 10.90$.

\section{Benzyl (2S)-3-methyl-2-(2-(4,4,5,5-tetramethyl-2-} (3-nitrophenyl)-4,5-dihydroimidazol-I-yloxy)acetamido) butanoate (5b)

Yield: $650 \mathrm{mg}(71 \%)$. ESI/MS (m/z) $511[\mathrm{M}+\mathrm{H}]^{+} .{ }^{1} \mathrm{H}$ NMR (300 MHz, DMSO- $\left.d_{6}\right): \delta / p p m=8.47(\mathrm{~m}, 1 \mathrm{H}), 8.35$ (dd, $J=8.4 \mathrm{~Hz}, J=2.1 \mathrm{~Hz}, 1 \mathrm{H}), 8.21$ (d, $J=8.1 \mathrm{~Hz}, 1 \mathrm{H}), 8.15$ (d, $J=7.8 \mathrm{~Hz}, 1 \mathrm{H}), 7.74(\mathrm{t}, J=7.8 \mathrm{~Hz}, 1 \mathrm{H}), 7.36(\mathrm{~m}, 5 \mathrm{H}), 5.13$ (s, 2H), $4.58(\mathrm{~s}, 2 \mathrm{H}), 4.23$ (dd, $J=8.1 \mathrm{~Hz}, J=6.3 \mathrm{~Hz}, 1 \mathrm{H})$, $2.06(\mathrm{~m}, 1 \mathrm{H}), 1.23(\mathrm{~s}, 6 \mathrm{H}), 1.11(\mathrm{~s}, 6 \mathrm{H}), 0.83$ (d, $J=6.6 \mathrm{~Hz}$, $3 \mathrm{H}), 0.82(\mathrm{~d}, J=6.6 \mathrm{~Hz}, 3 \mathrm{H}) .{ }^{13} \mathrm{C}$ NMR $\left(75 \mathrm{MHz}\right.$, DMSO- $d_{6}$ ): $\delta / \mathrm{ppm}=171.48,167.72,162.58,147.99,136.23,134.91$, $130.50,128.88,128.60,128.54,123.34,75.57,73.24,68.05$, 66.49, 57.56, 30.29, 23.72, 19.78, 19.30, 18.48. Anal. Calcd for $\mathrm{C}_{27} \mathrm{H}_{34} \mathrm{~N}_{4} \mathrm{O}_{6}: \mathrm{C}, 63.51 ; \mathrm{H}, 6.71 ; \mathrm{N}, 10.97$. Found: $\mathrm{C}, 63.30$; $\mathrm{H}, 6.55 ; \mathrm{N}, 10.76$.

\section{Benzyl (2S)-4-methyl-2-(2-(4,4,5,5-tetramethyl-2-} (3-nitrophenyl)-4,5-dihydroimidazol- I-yloxy)acetamido) pentanoate $(5 \mathrm{c})$

Yield: $500 \mathrm{mg}(65 \%)$. ESI/MS (m/z) $525[\mathrm{M}+\mathrm{H}]^{+} .{ }^{1} \mathrm{H}$ NMR (300 MHz, DMSO- $\left.d_{6}\right): \delta / p p m=8.42(\mathrm{~s}, 1 \mathrm{H}), 8.32(\mathrm{~d}, J=8.4$ $\mathrm{Hz}, 1 \mathrm{H}), 8.25$ (d, $J=7.5 \mathrm{~Hz}, 1 \mathrm{H}), 8.11$ (d, $J=7.5 \mathrm{~Hz}, 1 \mathrm{H})$, $7.71(\mathrm{t}, J=7.8 \mathrm{~Hz}, 1 \mathrm{H}), 7.34(\mathrm{~m}, 5 \mathrm{H}), 5.10(\mathrm{~s}, 2 \mathrm{H}), 4.49$ (s, 2H), $4.35(\mathrm{~m}, 1 \mathrm{H}), 1.56(\mathrm{~m}, 3 \mathrm{H}), 1.20(\mathrm{~s}, 6 \mathrm{H}), 1.12$ (s, 6H), 0.85 (d, $J=3.9 \mathrm{~Hz}, 3 \mathrm{H}), 0.80$ (d, $J=4.2 \mathrm{~Hz}, 3 \mathrm{H})$. ${ }^{13} \mathrm{C}$ NMR (75 MHz, DMSO- $d_{6}$ ): $\delta / \mathrm{ppm}=172.38,167.67$, $162.44,147.99,136.33,134.74,132.89,130.41,128.87$, $128.51,128.25,125.17,123.11,75.54,72.92,68.35,66.45$, 50.52, 24.64, 23.88, 23.12, 21.65, 19.83. Anal. Calcd for $\mathrm{C}_{28} \mathrm{H}_{36} \mathrm{~N}_{4} \mathrm{O}_{6}: \mathrm{C}, 64.10 ; \mathrm{H}, 6.92 ; \mathrm{N}, 10.68$. Found: C, 63.89; $\mathrm{H}, 6.77 ; \mathrm{N}, 10.47$.

Benzyl (2S)-2-(2-(4,4,5,5-tetramethyl-2-(3-nitrophenyl)4,5-dihydroimidazol-I-yloxy)acetamido)propanoate (5d) Yield: $425 \mathrm{mg}$ (44\%). ESI/MS (m/z) $483[\mathrm{M}+\mathrm{H}]^{+} .{ }^{1} \mathrm{H}$ NMR (300 MHz, DMSO- $d_{6}$ ): $\delta / \mathrm{ppm}=8.42(\mathrm{t}, J=1.8 \mathrm{~Hz}, 1 \mathrm{H}), 8.32$ (d, $J=6.6 \mathrm{~Hz}, 2 \mathrm{H}), 8.11(\mathrm{~d}, J=7.8 \mathrm{~Hz}, 1 \mathrm{H}), 7.71$ (t, $J=8.1 \mathrm{~Hz}$, 1H), $7.34(\mathrm{~m}, 5 \mathrm{H}), 5.11(\mathrm{~s}, 2 \mathrm{H}), 4.50(\mathrm{~s}, 2 \mathrm{H}), 4.33(\mathrm{t}, J=7.2 \mathrm{~Hz}$, $1 \mathrm{H}), 1.28(\mathrm{~d}, J=7.2 \mathrm{~Hz}, 3 \mathrm{H}), 1.20(\mathrm{~s}, 6 \mathrm{H}), 1.12(\mathrm{~s}, 6 \mathrm{H})$. ${ }^{13} \mathrm{C}$ NMR (75 MHz, DMSO- $d_{6}$ ): $\delta / \mathrm{ppm}=172.47,167.35$, $162.42,147.98,136.37,134.73,132.80,130.46,128.87$, $128.48,128.17,125.22,123.10,75.64,72.92,68.33,66.43$, 47.83, 23.90, 17.31. Anal. Calcd for $\mathrm{C}_{25} \mathrm{H}_{30} \mathrm{~N}_{4} \mathrm{O}_{6}: \mathrm{C}, 62.23$; H, 6.27; N, 11.61. Found: C, 62.04; H, 6.11; N, 11.83 .
Benzyl (2S)-(2-(4,4,5,5-tetramethyl-2-(3-nitrophenyl)-4,5dihydroimidazol-1-yloxy)acetamido)acetate (5e)

Yield: $810 \mathrm{mg}(87 \%)$. ESI/MS (m/z) $469[\mathrm{M}+\mathrm{H}]^{+} .{ }^{1} \mathrm{H}$ NMR $\left(300 \mathrm{MHz}, \mathrm{DMSO}-d_{6}\right): \delta / \mathrm{ppm}=8.41(\mathrm{~m}, 1 \mathrm{H})$, 8.33 (m, 1H), 8.23 (t, $J=7.5 \mathrm{~Hz}, 1 \mathrm{H}), 8.10$ (d, $J=7.8 \mathrm{~Hz}$, $1 \mathrm{H}), 7.71(\mathrm{t}, J=7.8 \mathrm{~Hz}, 1 \mathrm{H}), 7.36(\mathrm{~m}, 5 \mathrm{H}), 5.11(\mathrm{~s}, 2 \mathrm{H})$, $4.53(\mathrm{~s}, 2 \mathrm{H}), 3.89$ (d, J=6.0 Hz, 2H), $1.22(\mathrm{~s}, 6 \mathrm{H}), 1.13$ (s, 6H). ${ }^{13} \mathrm{C}$ NMR $\left(75 \mathrm{MHz}, \mathrm{DMSO}-d_{6}\right): \delta / \mathrm{ppm}=169.82$, $167.88,162.37,148.05,136.29,134.64,132.78,130.49$, 128.86, 128.53, 128.39, 125.22, 123.09, 75.06, 72.95, 68.35, 66.38, 23.88, 19.87. Anal. Calcd for $\mathrm{C}_{24} \mathrm{H}_{28} \mathrm{~N}_{4} \mathrm{O}_{6}$ : C, 61.53; H, 6.02; N, 11.96. Found: C, 61.74; H, 6.18; $\mathrm{N}, 12.17$.

Benzyl (2S)-3-(4-hydroxyphenyl)-2-(2-(4,4,5,5-tetramethyl2-(3-nitrophenyl)-4,5-dihydroimidazol-I-yloxy)acetamido) propanoate (5f)

Yield: $952 \mathrm{mg}(83 \%)$. ESI/MS (m/z) $573[\mathrm{M}-\mathrm{H}]^{-}$. ${ }^{1} \mathrm{H}$ NMR $\left(300 \mathrm{MHz}, \mathrm{DMSO}-d_{6}\right): \delta / \mathrm{ppm}=9.22(\mathrm{~s}, 1 \mathrm{H}), 8.42$ (t, $J=1.9 \mathrm{~Hz}, 1 \mathrm{H}), 8.32$ (dd, $J=8.4 \mathrm{~Hz}, J=2.1 \mathrm{~Hz}, 1 \mathrm{H}), 8.14$ (d, $J=8.1 \mathrm{~Hz}, 1 \mathrm{H}), 8.07$ (d, $J=7.5 \mathrm{~Hz}, 1 \mathrm{H}), 7.69$ (t, $J=8.1 \mathrm{~Hz}$, $1 \mathrm{H}), 7.34(\mathrm{~m}, 3 \mathrm{H}), 7.29(\mathrm{~m}, 2 \mathrm{H}), 6.93(\mathrm{~d}, J=8.4 \mathrm{~Hz}, 2 \mathrm{H}), 6.62$ (d, $J=6.5 \mathrm{~Hz}, 2 \mathrm{H}), 5.08(\mathrm{~m}, 2 \mathrm{H}), 4.52(\mathrm{~m}, 1 \mathrm{H}), 4.45(\mathrm{~s}, 2 \mathrm{H})$, 2.93 (dd, $J=14.0 \mathrm{~Hz}, J=6.0 \mathrm{~Hz}, 1 \mathrm{H}), 2.84$ (dd, $J=13.5 \mathrm{~Hz}$, $J=8.4 \mathrm{~Hz}, 1 \mathrm{H}), 1.15(\mathrm{~s}, 6 \mathrm{H}), 1.12(\mathrm{~m}, 6 \mathrm{H}),{ }^{13} \mathrm{C} \mathrm{NMR}$ $\left(75 \mathrm{MHz}, \mathrm{DMSO}-d_{6}\right): \delta / \mathrm{ppm}=171.51,167.43,162.40$, $156.59,148.02,136.15,134.63,132.80,130.45,128.83$, $128.51,128.34,127.08,125.19,123.09,115.55,75.45$, 72.92, 68.32, 66.55, 53.94, 36.26, 23.87, 14.55. Anal. Calcd for $\mathrm{C}_{31} \mathrm{H}_{34} \mathrm{~N}_{4} \mathrm{O}_{7}: \mathrm{C}, 64.80 ; \mathrm{H}, 5.96 ; \mathrm{N}, 9.75$. Found: $\mathrm{C}, 64.61$; $\mathrm{H}, 5.80 ; \mathrm{N}, 9.52$.

\section{Benzyl (2S)-dibenzyl-2-(2-(4,4,5,5-tetramethyl-2-}

(3-nitrophenyl)-4,5-dihydroimidazol-I-yloxy)acetamido) pentanedioate $(5 \mathrm{~g})$

Yield: $600 \mathrm{mg}(69 \%)$. ESI/MS (m/z) $631[\mathrm{M}+\mathrm{H}]^{+} .{ }^{1} \mathrm{H}$ NMR (300 MHz, DMSO- $\left.d_{6}\right): \delta / \mathrm{ppm}=8.42(\mathrm{~s}, 1 \mathrm{H}), 8.30(\mathrm{~m}$, 2H), 8.09 (d, $J=7.2 \mathrm{~Hz}, 1 \mathrm{H}), 7.68(\mathrm{t}, J=7.8 \mathrm{~Hz}, 1 \mathrm{H}), 7.34$ $(\mathrm{m}, 10 \mathrm{H}), 5.11(\mathrm{~s}, 2 \mathrm{H}), 5.07(\mathrm{~s}, 2 \mathrm{H}), 4.49(\mathrm{~s}, 2 \mathrm{H}), 4.39$ $(\mathrm{m}, 1 \mathrm{H}), 2.39(\mathrm{t}, J=7.5 \mathrm{~Hz}, 2 \mathrm{H}), 2.07(\mathrm{~m}, 1 \mathrm{H}), 1.91(\mathrm{~m}, 1 \mathrm{H})$, $1.19(\mathrm{~s}, 6 \mathrm{H}), 1.12(\mathrm{~s}, 6 \mathrm{H}) .{ }^{13} \mathrm{C}$ NMR (75 MHz, DMSO- $\left.d_{6}\right)$ : $\delta / \mathrm{ppm}=172.37,171.53,167.82,162.39,148.00,136.54$, $136.26,134.69,132.85,130.39,128.66,128.52,128.47$, $128.37,128.26,125.16,123.12,75.58,72.92,68.33,66.60$, $66.01,51.40,30.20,26.37,23.87,19.80$. Anal. Calcd for $\mathrm{C}_{34} \mathrm{H}_{38} \mathrm{~N}_{4} \mathrm{O}_{8}: \mathrm{C}, 64.75 ; \mathrm{H}, 6.07 ; \mathrm{N}, 8.88$. Found: C, 64.54; $\mathrm{H}, 5.91 ; \mathrm{N}, 8.67$. 
Benzyl (2S)-6-(tert-butoxycarbonyl)-2-(2-(4,4,5,5tetramethyl-2-(3-nitrophenyl)-4,5-dihydroimidazolI-yloxy)acetamido)hexanoate (5h)

Yield: $1,100 \mathrm{mg}(86 \%)$. ESI/MS (m/z) $640[\mathrm{M}+\mathrm{H}]^{+} .{ }^{1} \mathrm{H}$ NMR (300 MHz, DMSO- $\left.d_{6}\right): \delta / p p m=8.43(\mathrm{t}, J=4.8 \mathrm{~Hz}, 1 \mathrm{H}), 8.32$ (dd, $J=7.5 \mathrm{~Hz}, J=1.5 \mathrm{~Hz}, 1 \mathrm{H}), 8.23$ (d, $J=7.5 \mathrm{~Hz}, 1 \mathrm{H}), 8.10$ (d, $J=7.8 \mathrm{~Hz}, 1 \mathrm{H}), 7.71(\mathrm{t}, J=8.1 \mathrm{~Hz}, 1 \mathrm{H}), 7.36(\mathrm{~m}, 5 \mathrm{H})$, $6.73(\mathrm{t}, J=5.1 \mathrm{~Hz}, 1 \mathrm{H}), 5.11(\mathrm{~s}, 2 \mathrm{H}), 5.00(\mathrm{~s}, 2 \mathrm{H}), 4.28(\mathrm{~m}$, 1H), 2.85 (dd, $J=12.6 \mathrm{~Hz}, J=6.3 \mathrm{~Hz}, 2 \mathrm{H}), 1.78$ (m, 2H), 1.68 $(\mathrm{m}, 2 \mathrm{H}), 1.36(\mathrm{~s}, 9 \mathrm{H}), 1.31(\mathrm{~m}, 2 \mathrm{H}), 1.21(\mathrm{~s}, 6 \mathrm{H}), 1.23(\mathrm{~s}$, $6 \mathrm{H}) .{ }^{13} \mathrm{C}$ NMR (75 MHz, DMSO- $d_{6}$ ): $\delta / \mathrm{ppm}=172.02,167.70$, $162.43,156.01,148.01,136.33,134.71,132.87,130.42$, $128.86,128.50,128.25,125.18,123.12,77.79,75.61,72.93$, 68.34, 66.44, 52.19, 30.90, 29.48, 28.71, 23.03, 21.21. Anal. Calcd for $\mathrm{C}_{33} \mathrm{H}_{45} \mathrm{~N}_{5} \mathrm{O}_{8}: \mathrm{C}, 61.96 ; \mathrm{H}, 7.09 ; \mathrm{N}, 10.95$. Found: C, 61.76; H, 7.02; N, 10.78.

Benzyl (2S)-4-amino-4-oxo-2-(2-(4,4,5,5-tetramethyl2-(3-nitrophenyl)-4,5-dihydroimidazol-I-yloxy)acetamido) butanoate $(5 \mathrm{i})$

Yield: $852 \mathrm{mg}(74 \%)$. ESI/MS (m/z) $526[\mathrm{M}+\mathrm{H}]^{+} .{ }^{1} \mathrm{H}$ NMR (300 MHz, DMSO- $\left.d_{6}\right): \delta / p p m=8.41(\mathrm{~m}, 1 \mathrm{H}), 8.31$ (m, 1H), 8.18 (d, $J=8.1 \mathrm{~Hz}, 1 \mathrm{H}), 8.08$ (d, $J=7.8 \mathrm{~Hz}, 1 \mathrm{H})$, $7.71(\mathrm{t}, J=8.1 \mathrm{~Hz}, 1 \mathrm{H}), 7.42(\mathrm{~s}, 1 \mathrm{H}), 7.33(\mathrm{~m}, 5 \mathrm{H}), 6.95$ (s, 1H), 5.09 (s, 2H), 4.71 (dd, J=13.72 Hz, J=5.4 Hz, 1H), 4.51 (s, 2H), 2.59 (t, J=4.5 Hz, 2H), 1.19 (s, 6H), 1.12 (s, 6H). ${ }^{13} \mathrm{C}$ NMR (75 MHz, DMSO- $d_{6}$ ): $\delta / \mathrm{ppm}=171.54,171.23$, 167.32 , 162.44, 148.04, 136.36, 134.58, 132.75, 130.52, $128.79,128.36,128.00,125.23,123.03,75.72,72.94,68.34$, 66.52, 48.80, 36.65, 25.76, 24.92, 23.88, 15.62. Anal. Calcd for $\mathrm{C}_{26} \mathrm{H}_{31} \mathrm{~N}_{5} \mathrm{O}_{7}: \mathrm{C}, 59.42 ; \mathrm{H}, 5.95 ; \mathrm{N}, 13.33$. Found: $\mathrm{C}, 59.20$; $\mathrm{H}, 5.70 ; \mathrm{N}, 13.11$.

Benzyl (2S)-5-amino-5-oxo-2-(2-(4,4,5,5-tetramethyl2-(3-nitrophenyl)-4,5-dihydroimidazol-I-yloxy)acetamido) pentanoate $(5 \mathrm{j})$

Yield: $832 \mathrm{mg}(85 \%)$. ESI/MS (m/z) $540[\mathrm{M}+\mathrm{H}]^{+} .{ }^{1} \mathrm{H}$ NMR (300 MHz, DMSO- $\left.d_{6}\right): \delta / p p m=8.42(\mathrm{~m}, 1 \mathrm{H}), 8.37$ (m, 2H), $8.01(\mathrm{~d}, J=7.8 \mathrm{~Hz}, 1 \mathrm{H}), 7.71(\mathrm{t}, J=8.1 \mathrm{~Hz}, 1 \mathrm{H}), 7.35$ (m, 5H), $7.24(\mathrm{~s}, 1 \mathrm{H}), 6.77(\mathrm{~s}, 1 \mathrm{H}), 5.11(\mathrm{~s}, 2 \mathrm{H}), 4.49(\mathrm{~s}, 2 \mathrm{H})$, $4.32(\mathrm{~m}, 1 \mathrm{H}), 2.09(\mathrm{~m}, 2 \mathrm{H}), 1.96(\mathrm{~m}, 1 \mathrm{H}), 1.81(\mathrm{~m}, 1 \mathrm{H})$, $1.21(\mathrm{~s}, 6 \mathrm{H}), 1.09$ (s, 6H). ${ }^{13} \mathrm{C}$ NMR (75 MHz, DMSO- $d_{6}$ ): $\delta / \mathrm{ppm}=173.65,171.83,167.74,162.41,148.02,136.33$, $134.69,132.85,130.43,128.86,128.49,128.24,125.18$, $123.13,75.62,72.94,68.33,66.48,52.00,31.44,26.89$, 23.91, 19.84. Anal. Calcd for $\mathrm{C}_{27} \mathrm{H}_{33} \mathrm{~N}_{5} \mathrm{O}_{7}: \mathrm{C}, 60.10 ; \mathrm{H}, 6.16$; N, 12.98. Found: C, 60.29; H, 6.00; N, 12.75.
Benzyl (2S)-3-hydroxy-2-(2-(4,4,5,5-tetramethyl-2(3-nitrophenyl)-4,5-dihydroimidazol- I-yloxy)acetamido) butanoate $(5 \mathrm{k})$

Yield: $700 \mathrm{mg}(70 \%)$. ESI/MS (m/z) $513[\mathrm{M}+\mathrm{H}]^{+}$. ${ }^{1} \mathrm{H}$ NMR $\left(300 \mathrm{MHz}, \mathrm{DMSO}-d_{6}\right): \delta / \mathrm{ppm}=8.43(\mathrm{~m}, 1 \mathrm{H})$, 8.32 (dd, $J=8.1 \mathrm{~Hz}, J=1.5 \mathrm{~Hz}, 1 \mathrm{H}), 8.12$ (d, $J=7.8 \mathrm{~Hz}$, $1 \mathrm{H}), 7.82(\mathrm{~d}, J=7.6 \mathrm{~Hz}, 1 \mathrm{H}), 7.72(\mathrm{t}, J=7.8 \mathrm{~Hz}, 1 \mathrm{H}), 7.36$ $(\mathrm{m}, 5 \mathrm{H}), 5.13(\mathrm{~m}, 2 \mathrm{H}), 4.62(\mathrm{~s}, 2 \mathrm{H}), 4.37(\mathrm{dd}, J=8.1 \mathrm{~Hz}$, $J=3.9 \mathrm{~Hz}, 1 \mathrm{H}), 4.17(\mathrm{~m}, 1 \mathrm{H}), 1.21$ (s, 6H), 1.13 (s, 6H), $1.02(\mathrm{~d}, J=6.3 \mathrm{~Hz}, 3 \mathrm{H}) .{ }^{13} \mathrm{C}$ NMR $\left(75 \mathrm{MHz}\right.$, DMSO- $d_{6}$ ): $\delta / \mathrm{ppm}=170.62,168.07,162.51,148.03,136.33,134.63$, $132.77,130.52,128.83,128.44,128.16,125.24,123.06$, $75.55,72.95,68.37,66.55,57.82,23.91,20.64$. Anal. Calcd for $\mathrm{C}_{26} \mathrm{H}_{32} \mathrm{~N}_{4} \mathrm{O}_{7}: \mathrm{C}, 60.93 ; \mathrm{H}, 6.29 ; \mathrm{N}, 10.93$. Found: C, 60.70; H, 6.12; N, 10.71 .

Benzyl (2S)-3-(tert-butylthio)-2-(2-(4,4,5,5-tetramethyl2-(3-nitrophenyl)-4,5-dihydroimidazol-I-yloxy)acetamido) propanoate (5I)

Yield: $470 \mathrm{mg}$ (42\%). ESI/MS (m/z) 569 [M-H] ${ }^{-} .{ }^{1} \mathrm{H}$ NMR $\left(300 \mathrm{MHz}, \mathrm{DMSO}-d_{6}\right): \delta / \mathrm{ppm}=8.42(\mathrm{t}, J=1.8 \mathrm{~Hz}, 1 \mathrm{H}), 8.32$ (dd, $J=8.1 \mathrm{~Hz}, J=1.5 \mathrm{~Hz}, 1 \mathrm{H}), 8.24$ (d, $J=8.1 \mathrm{~Hz}, 1 \mathrm{H}), 8.11$ (d, $J=7.8 \mathrm{~Hz}, 1 \mathrm{H}), 7.71(\mathrm{t}, J=7.8 \mathrm{~Hz}, 1 \mathrm{H}), 7.36(\mathrm{~m}, 5 \mathrm{H})$, $5.12(\mathrm{~s}, 2 \mathrm{H}), 4.53(\mathrm{~m}, 1 \mathrm{H}), 4.52(\mathrm{~s}, 2 \mathrm{H}), 2.87(\mathrm{~m}, 1 \mathrm{H}), 2.76$ (m, 1H), $1.26(\mathrm{~s}, 6 \mathrm{H}), 1.22(\mathrm{~s}, 9 \mathrm{H}), 1.13(\mathrm{~s}, 6 \mathrm{H}) .{ }^{13} \mathrm{C}$ NMR (75 MHz, DMSO- $d_{6}$ ): $\delta / \mathrm{ppm}=170.44,167.53,162.44$, $148.03,136.13,134.67,132.84,130.49,128.84,128.54$, $128.31,128.27,125.21,123.06,75.49,72.96,68.38,66.80$, 52.77, 42.73, 31.14, 29.68, 23.90, 19.86. Anal. Calcd for $\mathrm{C}_{29} \mathrm{H}_{38} \mathrm{~N}_{4} \mathrm{O}_{6} \mathrm{~S}: \mathrm{C}, 61.03 ; \mathrm{H}, 6.71 ; \mathrm{N}, 9.82$. Found: C, 61.24; H, 6.88; N, 9.97.

Benzyl (2S)-4-(methylthio)-2-(2-(4,4,5,5-tetramethyl2-(3-nitrophenyl)-4,5-dihydroimidazol- I-yloxy)acetamido) butanoate $(5 \mathrm{~m})$

Yield: $842 \mathrm{mg}$ (78\%). ESI/MS (m/z) $543[\mathrm{M}+\mathrm{H}]^{+} .{ }^{1} \mathrm{H}$ NMR $\left(300 \mathrm{MHz}\right.$, DMSO- $\left.d_{6}\right): \delta / \mathrm{ppm}=8.43(\mathrm{~m}, 1 \mathrm{H}), 8.32(\mathrm{~d}, J=7.2$ $\mathrm{Hz}, 2 \mathrm{H}), 8.11$ (d, $J=8.1 \mathrm{~Hz}, 1 \mathrm{H}), 7.71$ (t, $J=8.1 \mathrm{~Hz}, 1 \mathrm{H})$, $7.35(\mathrm{~m}, 5 \mathrm{H}), 5.12(\mathrm{~s}, 2 \mathrm{H}), 4.55(\mathrm{~s}, 2 \mathrm{H}), 4.47(\mathrm{~m}, 1 \mathrm{H}), 2.42$ (m, 2H), $1.99(\mathrm{~s}, 3 \mathrm{H}), 1.93(\mathrm{~m}, 2 \mathrm{H}), 1.21(\mathrm{~s}, 6 \mathrm{H}), 1.13$ (s, 6H). ${ }^{13} \mathrm{C}$ NMR (75 MHz, DMSO- $\left.d_{6}\right): \delta / \mathrm{ppm}=171.68$, $167.81,162.41,148.00,136.30,134.72,132.86,130.41$, $128.87,128.54,128.30,125.18,123.12,75.60,72.93,68.34$, 66.59, 51.21, 30.75, 29.88, 23.89, 14.94. Anal. Calcd for $\mathrm{C}_{27} \mathrm{H}_{34} \mathrm{~N}_{4} \mathrm{O}_{6} \mathrm{~S}: \mathrm{C}, 59.76 ; \mathrm{H}, 6.32 ; \mathrm{N}, 10.32$. Found: $\mathrm{C}, 59.55$; $\mathrm{H}, 6.16 ; \mathrm{N}, 10.10$. 
Benzyl (2S)-5-(3-nitroguanidino)-2-(2-(4,4,5,5-tetramethyl2-(3-nitrophenyl)-4,5-dihydroimidazol- I-yloxy)acetamido) pentanoate $(5 n)$

Yield: 1,102 mg (90\%). ESI/MS (m/z) $613[\mathrm{M}+\mathrm{H}]^{+} .{ }^{1} \mathrm{H}$ NMR $\left(300 \mathrm{MHz}, \mathrm{DMSO}-d_{6}\right): \delta / \mathrm{ppm}=8.48(\mathrm{~m}, 1 \mathrm{H}), 8.43(\mathrm{~m}, 1 \mathrm{H})$, $8.33(\mathrm{~m}, 2 \mathrm{H}), 8.11(\mathrm{~d}, J=7.8 \mathrm{~Hz}, 1 \mathrm{H}), 7.96(\mathrm{~m}, 2 \mathrm{H}), 7.71$ (t, $J=7.8 \mathrm{~Hz}, 1 \mathrm{H}), 7.35(\mathrm{~m}, 5 \mathrm{H}), 5.11(\mathrm{~s}, 2 \mathrm{H}), 4.51(\mathrm{~s}, 2 \mathrm{H}), 4.34$ (m, 1H), 3.12 (dd, $J=12.3 \mathrm{~Hz}, J=6.3 \mathrm{~Hz}, 2 \mathrm{H}), 1.76(\mathrm{~m}, 1 \mathrm{H})$, $1.64(\mathrm{~m}, 1 \mathrm{H}), 1.47(\mathrm{~m}, 2 \mathrm{H}), 1.20(\mathrm{~s}, 6 \mathrm{H}), 1.12(\mathrm{~s}, 6 \mathrm{H}) .{ }^{13} \mathrm{C} \mathrm{NMR}$ (75 MHz, DMSO- $\left.d_{6}\right): \delta / p p m=171.86,167.76,162.41,148.02$, $136.27,134.69,132.85,130.43,128.87,128.52,128.27$, $125.18,123.12,79.64,75.61,72.91,68.34,66.55,51.95$, 28.43, 23.90, 19.84. Anal. Calcd for $\mathrm{C}_{28} \mathrm{H}_{36} \mathrm{~N}_{8} \mathrm{O}_{8}$ : C, 54.89; H, 5.92; N, 18.29. Found: C, 54.67; H, 5.75; N, 18.07.

Benzyl (2S)- I-(2-(4,4,5,5-tetramethyl-2-(3-nitrophenyl)4,5-dihydroimidazol- I-yloxy)acetyl)pyrrolidine-

2-carboxylate (5o)

Yield: $500 \mathrm{mg}$ (53\%). ESI/MS (m/z) $509[\mathrm{M}+\mathrm{H}]^{+} .{ }^{1} \mathrm{H}$ NMR $\left(300 \mathrm{MHz}, \mathrm{DMSO}-d_{6}\right): \delta / \mathrm{ppm}=8.43(\mathrm{~m}, 1 \mathrm{H}), 8.32$ (d, $J=8.1$ $\mathrm{Hz}, 1 \mathrm{H}), 8.11$ (d, $J=7.8 \mathrm{~Hz}, 1 \mathrm{H}), 7.71$ (t, $J=8.1 \mathrm{~Hz}, 1 \mathrm{H})$, $7.34(\mathrm{~m}, 5 \mathrm{H}), 5.11(\mathrm{~m}, 2 \mathrm{H}), 4.73(\mathrm{~s}, 2 \mathrm{H}), 4.35(\mathrm{~m}, 1 \mathrm{H}), 3.50$ $(\mathrm{m}, 2 \mathrm{H}), 2.16(\mathrm{~m}, 1 \mathrm{H}), 1.89(\mathrm{~m}, 3 \mathrm{H}), 1.21(\mathrm{~s}, 6 \mathrm{H}), 1.09(\mathrm{~s}$, $6 \mathrm{H}) .{ }^{13} \mathrm{C} \mathrm{NMR}\left(75 \mathrm{MHz}, \mathrm{DMSO}-d_{6}\right): \delta / \mathrm{ppm}=172.07,166.23$, $162.49,148.00,136.42,134.69,132.85,132.73,130.44$, $128.94,128.72,128.45,125.19,123.16,75.31,73.05,68.30$, 66.28, 58.93, 46.06, 28.90, 24.90, 23.92. Anal. Calcd for $\mathrm{C}_{27} \mathrm{H}_{32} \mathrm{~N}_{4} \mathrm{O}_{6}: \mathrm{C}, 63.77 ; \mathrm{H}, 6.34 ; \mathrm{N}, 11.02$. Found: C, 63.54; H, 6.20; N, 10.81 .

General procedure of preparing I-(4,4,5,5-tetramethyl-2(3-nitrophenyl)-4,5-dihydroimidazol- I-yloxy)acetyl-

L-amino acids (6a-o)

At $0^{\circ} \mathrm{C}$, to a solution of $1 \mathrm{mmol}$ of $5 \mathrm{a}-\mathrm{O}$ in $20 \mathrm{~mL}$ of methanol $2 \mathrm{~mL}$ of aqueous $\mathrm{NaOH}(4 \mathrm{M})$ was added. The reaction mixture was stirred for 1 hour, and TLC (EtOAC:petroleum=1:1) indicated the complete disappearance of $5 \mathrm{a}-\mathrm{O}$. The reaction mixture was adjusted to $\mathrm{pH} 2$ and evaporated under vacuum to remove methanol and water. The residue was purified with column chromatography $\left(\mathrm{CH}_{2} \mathrm{Cl}_{2}: \mathrm{CH}_{3} \mathrm{OH}=5: 1\right)$ to provide $6 \mathrm{a}-\mathrm{o}$.

(2S,3S)-3-methyl-2-(2-(4,4,5,5-tetramethyl-2-(3nitrophenyl)-4,5-dihydroimidazol- I-yloxy)acetamido) pentanoic acid (6a)

Yield: $320 \mathrm{mg}(72 \%)$. Mp $75^{\circ} \mathrm{C}-76^{\circ} \mathrm{C}$; ESI/MS (m/z) 433 $[\mathrm{M}-\mathrm{H}]^{-} \cdot[\alpha]_{20}{ }^{\mathrm{D}=} 0.3\left(\mathrm{c}=1.1, \mathrm{CH}_{3} \mathrm{OH}\right)$. IR (KBr): 3,413, 2,971, 2,905, 2,877, 1,682, 1,533, 1,451, 1,349, 1,214, 1,158, 1,062,
902, 823, 718, $573 \mathrm{~cm}^{-1} .{ }^{1} \mathrm{H}$ NMR (300 MHz, DMSO- $\left.d_{6}\right)$ : $\delta / \mathrm{ppm}=8.43(\mathrm{~m}, 1 \mathrm{H}), 8.32(\mathrm{dd}, J=8.1 \mathrm{~Hz}, J=1.5 \mathrm{~Hz}, 1 \mathrm{H}), 8.12$ $(\mathrm{d}, J=7.8 \mathrm{~Hz}, 1 \mathrm{H}), 7.81(\mathrm{~d}, J=8.4 \mathrm{~Hz}, 1 \mathrm{H}), 7.73(\mathrm{~d}, J=8.1 \mathrm{~Hz}$, $1 \mathrm{H}), 4.53(\mathrm{~s}, 2 \mathrm{H}), 4.17(\mathrm{~m}, 1 \mathrm{H}), 1.75(\mathrm{~m}, 1 \mathrm{H}), 1.40(\mathrm{~m}, 2 \mathrm{H})$, $1.22(\mathrm{~s}, 6 \mathrm{H}), 1.13(\mathrm{~s}, 6 \mathrm{H}), 0.81(\mathrm{~m}, 6 \mathrm{H}) .{ }^{13} \mathrm{C} \mathrm{NMR}(75 \mathrm{MHz}$, DMSO- $\left.d_{6}\right): \delta / \mathrm{ppm}=173.11,167.22,162.53,148.02,134.72$, 132.89, 130.46, 128.47, 125.17, 123.08, 75.62, 72.94, 68.37, 56.59, 37.11, 25.10, 23.89, 15.92, 11.77. Anal. Calcd for $\mathrm{C}_{21} \mathrm{H}_{30} \mathrm{~N}_{4} \mathrm{O}_{6}: \mathrm{C}, 58.05 ; \mathrm{H}, 6.96$; N, 12.89. Found: C, 57.84; $\mathrm{H}, 6.82 ; \mathrm{N}, 13.12$.

(2S)-3-methyl-2-(2-(4,4,5,5-tetramethyl-2-(3-nitrophenyl)4,5-dihydroimidazol-I-yloxy)acetamido)butanoic acid (6b) Yield: $450 \mathrm{mg}(78 \%)$. Mp $90^{\circ} \mathrm{C}-92^{\circ} \mathrm{C}$; ESI/MS (m/z) 419 $[\mathrm{M}-\mathrm{H}]^{-} .[\alpha]_{20}{ }^{\mathrm{D}=}-18.7\left(\mathrm{c}=1.2, \mathrm{CH}_{3} \mathrm{OH}\right) . \mathrm{IR}(\mathrm{KBr}): 3,210$, 2,970, 2,925, 2,870, 2,699, 1,734, 1,684, 1,536, 1,460, 1,352, $1,205,1,109,1,011,887,829,700 \mathrm{~cm}^{-1} ;{ }^{1} \mathrm{H}$ NMR $(300$ MHz, DMSO- $\left.d_{6}\right): \delta / p p m=12.90(\mathrm{~s}, 1 \mathrm{H}), 8.81(\mathrm{~m}, 1 \mathrm{H}), 8.50$ (dd, $J=8.4 \mathrm{~Hz}, J=1.5 \mathrm{~Hz}, 1 \mathrm{H}), 8.42$ (d, $J=8.1 \mathrm{~Hz}, 1 \mathrm{H}), 8.38$ (d, $J=8.2 \mathrm{~Hz}, 1 \mathrm{H}), 7.93$ (t, $J=8.1 \mathrm{~Hz}, 1 \mathrm{H}), 4.84$ (s, 2H), 4.09 (dd, $J=5.7 \mathrm{~Hz}, J=8.4 \mathrm{~Hz}, 1 \mathrm{H}), 2.03$ (m, 1H), 1.47 (s, 6H), 1.42 (s, $6 \mathrm{H}), 0.85$ (d, $J=6.6 \mathrm{~Hz}, 6 \mathrm{H}) .{ }^{13} \mathrm{C} \mathrm{NMR}\left(75 \mathrm{MHz}, \mathrm{DMSO}-d_{6}\right): \delta /$ $\mathrm{ppm}=172.82$, 166.16, 163.29, 147.95, 136.45, 131.11, 128.90, 127.04, 125.37, 76.77, 75.91, 65.55, 63.29, 57.45, 30.23, 22.12, 19.44, 18.32, 15.63. Anal. Calcd for $\mathrm{C}_{20} \mathrm{H}_{28} \mathrm{~N}_{4} \mathrm{O}_{6}: \mathrm{C}, 57.13 ; \mathrm{H}$, $6.71 ; \mathrm{N}, 13.33$. Found: C, 57.35; H, 6.88; N, 13.56.

(2S)-4-methyl-2-(2-(4,4,5,5-tetramethyl-2-

(3-nitrophenyl)-4,5-dihydroimidazol- I-yloxy)acetamido) pentanoic acid (6c)

Yield: $401 \mathrm{mg}(82 \%) . \mathrm{Mp} 112^{\circ} \mathrm{C}-120^{\circ} \mathrm{C}$; ESI/MS (m/z) $433[\mathrm{M}-\mathrm{H}]^{-} \cdot[\alpha]_{20}{ }^{\mathrm{D}=}=44.9\left(\mathrm{c}=1.2, \mathrm{CH}_{3} \mathrm{OH}\right) . \mathrm{IR}(\mathrm{KBr}): 3,800$, $3,216,2,959,2,680,2,694,1,682,1,624,1,585,1,448,1,351$, 1,202, 1,160, 1,103, 902, 825, $700 \mathrm{~cm}^{-1}$; ${ }^{1} \mathrm{H}$ NMR (300 MHz, DMSO- $\left.d_{6}\right): \delta / \mathrm{ppm}=8.61(\mathrm{~s}, 1 \mathrm{H}), 8.45(\mathrm{~d}, J=7.8 \mathrm{~Hz}, 1 \mathrm{H})$, 8.27 (t, J=7.5 Hz, 2H), 7.82 (t, J=7.8 Hz, 1H), $4.62(\mathrm{~s}, 2 \mathrm{H})$, $4.20(\mathrm{~m}, 1 \mathrm{H}), 1.50(\mathrm{~m}, 3 \mathrm{H}), 1.35(\mathrm{~s}, 6 \mathrm{H}), 1.27(\mathrm{~s}, 6 \mathrm{H}), 0.88$ $(\mathrm{d}, J=3.9 \mathrm{~Hz}, 3 \mathrm{H}), 0.81(\mathrm{~d}, J=4.2 \mathrm{~Hz}, 3 \mathrm{H}) .{ }^{13} \mathrm{C} \mathrm{NMR}(75 \mathrm{MHz}$, DMSO- $\left.d_{6}\right): \delta / \mathrm{ppm}=173.93,166.69,162.88,147.97,135.58$, $130.78,128.47,126.99,124.22,76.20,74.40$, 66.99, 65.38, 50.42, 42.71, 23.24, 23.01, 21.69, 19.62, 15.64. Anal. Calcd for $\mathrm{C}_{21} \mathrm{H}_{30} \mathrm{~N}_{4} \mathrm{O}_{6}$ : C, 58.05; H, 6.96; N, 12.89. Found: C, 58.24; $\mathrm{H}, 6.81 ; \mathrm{N}, 12.65$.

(2S)-2-(2-(4,4,5,5-tetramethyl-2-(3-nitrophenyl)-4,5dihydroimidazol-I-yloxy)acetamido)propanoic acid (6d) Yield: $270 \mathrm{mg}$ (20\%). Mp 80 $\mathrm{C}-90^{\circ} \mathrm{C}$; ESI/MS (m/z) 391 $[\mathrm{M}-\mathrm{H}]^{-} .[\alpha]_{20}{ }^{\mathrm{D}=}-2.7\left(\mathrm{c}=1.3, \mathrm{CH}_{3} \mathrm{OH}\right)$. IR (KBr): 3,404, 
2,980, 2,972, 1,672, 1,612, 1,532, 1,452, 1,351, 1,158, 1,059, 905, 823, 717, $573 \mathrm{~cm}^{-1}$; ${ }^{1} \mathrm{H}$ NMR (300 MHz, DMSO- $d_{6}$ ): $\delta / \mathrm{ppm}=8.39$ (s, 1H), $8.23(\mathrm{~d}, J=6.3 \mathrm{~Hz}, 1 \mathrm{H}), 8.19$ (d, $J=5.7$ $\mathrm{Hz}, 1 \mathrm{H}), 7.73$ (m, 2H), $4.48(\mathrm{~s}, 2 \mathrm{H}), 3.94(\mathrm{~m}, 1 \mathrm{H}), 1.23$ (m, 6H), $1.22(\mathrm{~s}, 3 \mathrm{H}), 1.13(\mathrm{~s}, 6 \mathrm{H}) .{ }^{13} \mathrm{C}$ NMR $(75 \mathrm{MHz}$, DMSO- $\left.d_{6}\right): \delta / \mathrm{ppm}=166.23,162.52,148.01,134.64,132.72$, $130.59,125.26,122.99,76.06,72.91,68.34,65.39,23.9$, 15.63. Anal. Calcd for $\mathrm{C}_{18} \mathrm{H}_{24} \mathrm{~N}_{4} \mathrm{O}_{6}: \mathrm{C}, 55.09 ; \mathrm{H}, 6.16 ; \mathrm{N}$, 14.28. Found: C, 55.28; H, 6.33; N, 14.51.

(2S)-2-(2-(4,4,5,5-tetramethyl-2-(3-nitrophenyl)-4,5dihydroimidazol- I-yloxy)acetamido)acetic acid (6e)

Yield: $400 \mathrm{mg}(54 \%) . \mathrm{Mp} 172^{\circ} \mathrm{C}-176^{\circ} \mathrm{C}$; ESI/MS (m/z) $377[\mathrm{M}-\mathrm{H}]^{-} .[\alpha]_{20} \mathrm{D}=-5.7\left(\mathrm{c}=1.2, \mathrm{CH}_{3} \mathrm{OH}\right)$. IR $(\mathrm{KBr}): 3,431$, $3,086,2,983,2,960,1,726,1,682,1,606,1,535,1,444,1,348$, $1,223,1,164,1,120,1,064,888,822,712,555 \mathrm{~cm}^{-1} ;{ }^{1} \mathrm{H}$ NMR $\left(300 \mathrm{MHz}, \mathrm{DMSO}-d_{6}\right): \delta / \mathrm{ppm}=8.41(\mathrm{~m}, 1 \mathrm{H}), 8.32(\mathrm{~d}, J=8.1 \mathrm{~Hz}$, $1 \mathrm{H}), 8.11(\mathrm{~d}, J=7.8 \mathrm{~Hz}, 1 \mathrm{H}), 7.86(\mathrm{~m}, 1 \mathrm{H}), 7.73(\mathrm{t}, J=7.8 \mathrm{~Hz}$, 1H), 4.50 (s, 2H), 3.68 (d, J=5.4 Hz, 2H), 1.23 (s, 6H), 1.15 (s, 6H). ${ }^{13} \mathrm{C}$ NMR (75 MHz, DMSO- $d_{6}$ ): $\delta / \mathrm{ppm}=171.50$, 167.32, 162.42, 148.04, 134.66, 132.76, 130.52, 125.23, 123.07, 75.79, 72.95, 68.34, 23.89, 21.58, 19.90. Anal. Calcd for $\mathrm{C}_{17} \mathrm{H}_{22} \mathrm{~N}_{4} \mathrm{O}_{6}: \mathrm{C}, 53.96 ; \mathrm{H}, 5.86 ; \mathrm{N}, 14.81$. Found: $\mathrm{C}, 53.74$; $\mathrm{H}, 5.70 ; \mathrm{N}, 14.59$.

(2S)-3-(4-hydroxyphenyl)-2-(2-(4,4,5,5-tetramethyl2-(3-nitrophenyl)-4,5-dihydroimidazol- I-yloxy)acetamido) propanoic acid (6f)

Yield: $327 \mathrm{mg}(69 \%) . \mathrm{Mp} 79^{\circ} \mathrm{C}-82^{\circ} \mathrm{C}$; ESI/MS (m/z) 483 $[\mathrm{M}-\mathrm{H}]^{-} .[\alpha]_{20}^{\mathrm{D}=}=0.6\left(\mathrm{c}=1.2, \mathrm{CH}_{3} \mathrm{OH}\right)$. IR (KBr): 3,399, 2,980, 2,932, 1,672, 1,619, 1,532, 1,445, 1,351, 1,231, 1,166, $1,113,830,709,539 \mathrm{~cm}^{-1} ;{ }^{1} \mathrm{H}$ NMR (300 MHz, DMSO- $d_{6}$ ): $\delta / \mathrm{ppm}=12.74(\mathrm{~s}, 1 \mathrm{H}), 9.18(\mathrm{~s}, 1 \mathrm{H}), 8.42(\mathrm{~s}, 1 \mathrm{H}), 8.32(\mathrm{~d}, J=7.8 \mathrm{~Hz}$, $1 \mathrm{H}), 8.07(\mathrm{~d}, J=7.5 \mathrm{~Hz}, 1 \mathrm{H}), 7.86(\mathrm{~d}, J=7.5 \mathrm{~Hz}, 1 \mathrm{H}), 7.70(\mathrm{t}$, $J=7.8 \mathrm{~Hz}, 1 \mathrm{H}), 6.93(\mathrm{~d}, J=8.1 \mathrm{~Hz}, 2 \mathrm{H}), 6.61(\mathrm{~d}, J=8.1 \mathrm{~Hz}$, 2H), 4.45 (s, 2H), 4.39 (m, 1H), 2.93 (dd, $J=13.5 \mathrm{~Hz}, J=4.5$ $\mathrm{Hz}, 1 \mathrm{H}), 2.78(\mathrm{~m}, 1 \mathrm{H}), 1.15(\mathrm{~m}, 12 \mathrm{H}) .{ }^{13} \mathrm{C}$ NMR $(75 \mathrm{MHz}$, DMSO- $d_{6}$ ): $\delta / \mathrm{ppm}=173.00,167.12,162.48,156.46,148.02$, 134.65, 130.44, 127.52, 125.28, 123.13, 115.45, 75.53, 73.01, 68.25, 65.76, 53.06, 36.24, 23.84, 15.62. Anal. Calcd for $\mathrm{C}_{24} \mathrm{H}_{28} \mathrm{~N}_{4} \mathrm{O}_{7}: \mathrm{C}, 59.50 ; \mathrm{H}, 5.83 ; \mathrm{N}, 11.56$. Found: $\mathrm{C}, 59.72$; $\mathrm{H}, 5.99 ; \mathrm{N}, 11.78$.

(2S)-2-(2-(4,4,5,5-tetramethyl-2-(3-nitrophenyl)-4,5dihydroimidazol-I-yloxy)acetamido)pentanedioic acid (6g) Yield: $230 \mathrm{mg}(25 \%) . \mathrm{Mp} 54^{\circ} \mathrm{C}-58^{\circ} \mathrm{C}$; ESI/MS (m/z) 449 $[\mathrm{M}-\mathrm{H}]^{-} .[\alpha]_{20}{ }^{\mathrm{D}=}=-20.4\left(\mathrm{c}=1.2, \mathrm{CH}_{3} \mathrm{OH}\right) . \mathrm{IR}(\mathrm{KBr}): 3,330$, $3,112,2,984,2,865,1,740,1,541,1,357,1,250,1,026,906$,
824, 701, 636, $515 \mathrm{~cm}^{-1}$; ${ }^{1} \mathrm{H}$ NMR (300 MHz, DMSO- $d_{6}$ ): $\delta / \mathrm{ppm}=12.24(\mathrm{~m}, 2 \mathrm{H}), 8.67(\mathrm{~s}, 1 \mathrm{H}), 8.48(\mathrm{~d}, J=7.5 \mathrm{~Hz}, 1 \mathrm{H}), 8.38$ $(\mathrm{m}, 1 \mathrm{H}), 8.30(\mathrm{~m}, 1 \mathrm{H}), 7.86(\mathrm{~m}, 1 \mathrm{H}), 4.69(\mathrm{~s}, 2 \mathrm{H}), 4.20(\mathrm{~m}$, 1H), $2.23(\mathrm{~m}, 2 \mathrm{H}), 1.96(\mathrm{~m}, 1 \mathrm{H}), 1.77(\mathrm{~m}, 1 \mathrm{H}), 1.38(\mathrm{~s}, 6 \mathrm{H})$, $1.31(\mathrm{~s}, 6 \mathrm{H}) .{ }^{13} \mathrm{C}$ NMR $\left(75 \mathrm{MHz}, \mathrm{DMSO}-d_{6}\right): \delta / \mathrm{ppm}=174.03$, $173.11,172.40,166.59,163.05,148.00,135.82,130.93$, 124.57, 76.42, 74.92, 66.56, 65.39, 51.44, 30.45, 26.64, 22.78, 21.58, 19.56, 15.66. Anal. Calcd for $\mathrm{C}_{20} \mathrm{H}_{26} \mathrm{~N}_{4} \mathrm{O}_{8}: \mathrm{C}, 53.33 ; \mathrm{H}$, 5.82; N, 12.44. Found: C, 53.54; H, 5.97; N, 12.68.

(2S)-6-amino-2-(2-(4,4,5,5-tetramethyl-2(3-nitrophenyl)-4,5-dihydroim-idazol-I-yloxy)acetamido) hexanoic acid (6h)

Yield: $372 \mathrm{mg}$ (80\%). Mp $64^{\circ} \mathrm{C}-66^{\circ} \mathrm{C}$; ESI/MS (m/z) 448 $[\mathrm{M}-\mathrm{H}]^{-} \cdot[\alpha]_{20}^{\mathrm{D}=}=-21.2\left(\mathrm{c}=1.2, \mathrm{CH}_{3} \mathrm{OH}\right) . \mathrm{IR}(\mathrm{KBr}): 3,392,3,220$, 2,982, 2,723, 1,734, 1,684, 1,535, 1,352, 1,206, 1,159, 1,107, 1,020,883, 826, 698, $507 \mathrm{~cm}^{-1},{ }^{1} \mathrm{HNMR}\left(300 \mathrm{MHz}\right.$, DMSO- $\left.d_{6}\right)$ : $\delta / \mathrm{ppm}=8.82(\mathrm{~m}, 1 \mathrm{H}), 8.62(\mathrm{~m}, 2 \mathrm{H}), 8.43(\mathrm{~d}, J=8.1 \mathrm{~Hz}$, 1H), 8.14 (m, 2H), 7.94 (t, J=7.8 Hz, 1H), 4.78 (s, 2H), 4.11 (m, 1H), $2.71(\mathrm{~m}, 2 \mathrm{H}), 1.65(\mathrm{~m}, 1 \mathrm{H}), 1.56(\mathrm{~m}, 2 \mathrm{H}), 1.44$ $(\mathrm{m}, 12 \mathrm{H}), 1.34(\mathrm{~m}, 3 \mathrm{H}) \cdot{ }^{13} \mathrm{C}$ NMR (75 MHz, DMSO- $\left.d_{6}\right)$ : $\delta / \mathrm{ppm}=173.27,165.97,163.31,147.97,136.48,131.17$, $128.99,125.43,123.78,76.86,76.01,65.52,60.21,52.16$, $30.52,26.83,22.72,22.12,21.54,21.22,19.38,14.55$. Anal. Calcd for $\mathrm{C}_{21} \mathrm{H}_{31} \mathrm{~N}_{5} \mathrm{O}_{6}: \mathrm{C}, 56.11 ; \mathrm{H}, 6.95 ; \mathrm{N}, 15.58$. Found: C, 55.85; H, 6.87; N, 15.72 .

(2S)-4-amino-4-oxo-2-(2-(4,4,5,5-tetramethyl-2-(3nitrophenyl)-4,5-dihydroimidazol-I-yloxy)acetamido) butanoic acid (6i)

Yield: $326 \mathrm{mg}(51 \%) . \mathrm{Mp} 89^{\circ} \mathrm{C}-92^{\circ} \mathrm{C}$; ESI/MS (m/z) 436 $[\mathrm{M}+\mathrm{H}]+.[\alpha]_{20} \mathrm{D}=0.6\left(\mathrm{c}=1.3, \mathrm{CH}_{3} \mathrm{OH}\right) . \mathrm{IR}(\mathrm{KBr}): 3,370,3,230$, $3,111,2,982,2,850,1,737,1,678,1,540,1,450,1,357,1,244$, 1,170, 1,028, 906, 829, 702, 638, $517 \mathrm{~cm}^{-1} ;{ }^{1} \mathrm{H} N M R(300 \mathrm{MHz}$, DMSO- $\left.d_{6}\right): \delta / \mathrm{ppm}=9.48(\mathrm{~s}, 1 \mathrm{H}), 8.77(\mathrm{~s}, 1 \mathrm{H}), 8.61(\mathrm{~d}, J=8.1 \mathrm{~Hz}$, 1H), 8.32 (t, $J=6.9 \mathrm{~Hz}, 2 \mathrm{H}), 7.96$ (t, J=7.8 Hz, 1H), 7.38 $(\mathrm{s}, 1 \mathrm{H}), 6.90(\mathrm{~s}, 1 \mathrm{H}), 4.80(\mathrm{~s}, 2 \mathrm{H}), 4.50(\mathrm{~m}, 1 \mathrm{H}), 2.51(\mathrm{~m}, 2 \mathrm{H})$, $1.48(\mathrm{~s}, 6 \mathrm{H}), 1.41(\mathrm{~s}, 6 \mathrm{H}) .{ }^{13} \mathrm{C}$ NMR $\left(75 \mathrm{MHz}\right.$, DMSO- $\left.d_{6}\right)$ : $\delta / \mathrm{ppm}=172.64,171.62,165.56,163.83,148.11,136.13,131.39$, 129.16, 125.13, 123.58, 77.08, 76.24, 65.33, 48.82, 36.61, 22.14, 19.28, 15.61. Anal. Calcd for $\mathrm{C}_{19} \mathrm{H}_{25} \mathrm{~N}_{5} \mathrm{O}_{7}: \mathrm{C}, 52.41 ; \mathrm{H}$, 5.79; N, 16.08. Found: C, 52.20; H, 5.63; N, 15.84 .

(2S)-5-amino-5-oxo-2-(2-(4,4,5,5-tetramethyl-2-(3nitrophenyl)-4,5-dihydroimidazol-I-yloxy)acetamido) pentanoic acid (6j)

Yield: $378 \mathrm{mg}(74 \%) . \mathrm{Mp} 61^{\circ} \mathrm{C}-63^{\circ} \mathrm{C}$; ESI/MS (m/z) 448 $[\mathrm{M}-\mathrm{H}]^{-} .[\alpha]_{20}{ }^{\mathrm{D}=}=-13.7\left(\mathrm{c}=1.1, \mathrm{CH}_{3} \mathrm{OH}\right)$. IR $(\mathrm{KBr}): 3,413$, 
3,301, 2,950, 2,875, 1,739, 1,630, 1,541, 1,357, 1,285, 1,243, $1,169,1,028,637,516 \mathrm{~cm}^{-1} ;{ }^{1} \mathrm{H}$ NMR (300 MHz, DMSO- $d_{6}$ ): $\delta / \mathrm{ppm}=10.74(\mathrm{~m}, 1 \mathrm{H}), 8.77(\mathrm{~s}, 1 \mathrm{H}), 8.61(\mathrm{~d}, J=8.1 \mathrm{~Hz}, 1 \mathrm{H})$, $8.44(\mathrm{~d}, J=7.2 \mathrm{~Hz}, 1 \mathrm{H}), 8.34(\mathrm{~d}, J=7.5 \mathrm{~Hz}, 1 \mathrm{H}), 7.96(\mathrm{t}, J=8.1 \mathrm{~Hz}$, $1 \mathrm{H}), 7.23(\mathrm{~s}, 1 \mathrm{H}), 6.80(\mathrm{~s}, 1 \mathrm{H}), 4.77(\mathrm{~s}, 2 \mathrm{H}), 4.14(\mathrm{~m}, 1 \mathrm{H})$, $2.08(\mathrm{~m}, 2 \mathrm{H}), 1.94(\mathrm{~m}, 1 \mathrm{H}), 1.76(\mathrm{~m}, 1 \mathrm{H}), 1.49(\mathrm{~s}, 6 \mathrm{H}), 1.41$ $(\mathrm{s}, 6 \mathrm{H}) \cdot{ }^{13} \mathrm{C}$ NMR $\left(75 \mathrm{MHz}, \mathrm{DMSO}-d_{6}\right): \delta / \mathrm{ppm}=173.79$, 173.20, 165.84, 163.71, 148.07, 136.21, 131.31, 129.16, 125.20, 123.62, 76.94, 76.28, 65.47, 51.90, 31.57, 21.07, 22.11, 19.31, 15.61. Anal. Calcd for $\mathrm{C}_{20} \mathrm{H}_{27} \mathrm{~N}_{5} \mathrm{O}_{7}: \mathrm{C}, 53.45$; H, 6.05; N, 15.58. Found: C, 53.22; H, 6.23; N, 15.33.

(2S,3R)-3-hydroxy-2-(2-(4,4,5,5-tetramethyl-2-(3nitrophenyl)-4,5-dihydroimidazol- I-yloxy)acetamido) butanoic acid (6k)

Yield: $280 \mathrm{mg}$ (62\%). $\mathrm{Mp} 121^{\circ} \mathrm{C}-123^{\circ} \mathrm{C}$; ESI/MS (m/z) 421 $[\mathrm{M}-\mathrm{H}]^{-} .[\alpha]_{20} \mathrm{D}=-2.0\left(\mathrm{c}=1.2, \mathrm{CH}_{3} \mathrm{OH}\right) . \mathrm{IR}(\mathrm{KBr}): 3,406,2,979$, 2,920, 1,668, 1,611, 1,532, 1,408, 1,351, 1,159, 1,080, 811, $716,579 \mathrm{~cm}^{-1} ;{ }^{1} \mathrm{H}$ NMR (300 MHz, DMSO- $\left.d_{6}\right): \delta / \mathrm{ppm}=8.40$ (m, 1H), 8.32 (d, J=8.1 Hz, 1H), 8.09 (d, $J=7.5 \mathrm{~Hz}, 1 \mathrm{H})$, $7.74(\mathrm{t}, J=8.1 \mathrm{~Hz}, 1 \mathrm{H}), 7.43(\mathrm{~m}, 1 \mathrm{H}), 4.51(\mathrm{~s}, 2 \mathrm{H}), 3.89$ (m, 2H), $1.23(\mathrm{~s}, 6 \mathrm{H}), 1.13(\mathrm{~s}, 6 \mathrm{H}), 0.84(\mathrm{~m}, 3 \mathrm{H}) \cdot{ }^{13} \mathrm{C} \mathrm{NMR}$ $\left(75 \mathrm{MHz}, \mathrm{DMSO}-d_{6}\right): \delta / \mathrm{ppm}=166.40,162.53,148.04,134.56$, 132.68, 130.63, 125.27, 122.96, 76.03, 72.59, 68.32, 65.39, 23.88, 19.23, 15.64. Anal. Calcd for $\mathrm{C}_{19} \mathrm{H}_{26} \mathrm{~N}_{4} \mathrm{O}_{7}: \mathrm{C}, 54.02$; H, 6.20; N, 13.26. Found: C, 53.80; H, 6.13; N, 13.02.

(2S)-3-mercapto-2-(2-(4,4,5,5-tetramethyl-2-(3nitrophenyl)-4,5-dihydroimidazol-I-yloxy)acetamido) propanoic acid (6l)

Yield: $204 \mathrm{mg}(24 \%) . \mathrm{Mp} 117^{\circ} \mathrm{C}-118^{\circ} \mathrm{C}$; ESI/MS (m/z) 425 $[\mathrm{M}+\mathrm{H}]^{+} .[\alpha]_{20}{ }^{\mathrm{D}=}=-52.8\left(\mathrm{c}=1.3, \mathrm{CH}_{3} \mathrm{OH}\right) . \mathrm{IR}(\mathrm{KBr}): 3,381,3,240$, $3,105,2,944,1,743,1,668,1,540,1,451,1,356,1,289,1,240$, 1,170, 1,031, 829, 703, 637, $516 \mathrm{~cm}^{-1}$; ${ }^{1} \mathrm{H}$ NMR (300 MHz, DMSO- $\left.d_{6}\right): \delta / \mathrm{ppm}=12.10(\mathrm{~m}, 1 \mathrm{H}), 8.77(\mathrm{~s}, 1 \mathrm{H}), 8.60$ $(\mathrm{d}, J=8.1 \mathrm{~Hz}, 1 \mathrm{H}), 8.48(\mathrm{~d}, J=7.8 \mathrm{~Hz}, 1 \mathrm{H}), 8.32(\mathrm{~d}, J=7.8 \mathrm{~Hz}$, 1H), $7.93(\mathrm{~m}, 1 \mathrm{H}), 4.81(\mathrm{~s}, 2 \mathrm{H}), 4.40(\mathrm{~m}, 1 \mathrm{H}), 3.06(\mathrm{~m}, 1 \mathrm{H})$, $2.84(\mathrm{~m}, 1 \mathrm{H}), 1.48(\mathrm{~s}, 6 \mathrm{H}), 1.41(\mathrm{~s}, 6 \mathrm{H}) .{ }^{13} \mathrm{C} \mathrm{NMR}(75 \mathrm{MHz}$, DMSO- $\left.d_{6}\right): \delta / \mathrm{ppm}=171.82,165.97,163.76,148.09,136.14$, 131.33, 129.11, 125.15, 123.71, 76.88, 76.24, 65.55, 65.36, 22.18, 19.34, 15.62. Anal. Calcd for $\mathrm{C}_{18} \mathrm{H}_{24} \mathrm{~N}_{4} \mathrm{O}_{6} \mathrm{~S}: \mathrm{C}, 50.93$; H, 5.70; N, 13.20. Found: C, 50.70; H, 5.54; N, 13.01 .

(2S)-4-(methylthio)-2-(2-(4,4,5,5-tetramethyl-2-(3nitrophenyl)-4,5-dihydroimidazol- I-yloxy)acetamido) butanoic acid $(6 \mathrm{~m})$

Yield: $393 \mathrm{mg}$ (73\%). $\mathrm{Mp} 59^{\circ} \mathrm{C}-63^{\circ} \mathrm{C}$; ESI/MS (m/z) 451 $[\mathrm{M}-\mathrm{H}]^{-} \cdot[\alpha]_{20}{ }^{\mathrm{D}=} 1.0\left(\mathrm{c}=1.1, \mathrm{CH}_{3} \mathrm{OH}\right)$. IR (KBr): 3,406, 2,978,
2,923, 1,679, 1,533, 1,442, 1,350, 1,228, 1,163, 1,117, $1,063,714,570 \mathrm{~cm}^{-1} ;{ }^{1} \mathrm{H}$ NMR (300 MHz, DMSO- $\left.d_{6}\right): \delta /$ ppm=8.43 (m, 1H), $8.33(\mathrm{~d}, J=6.9 \mathrm{~Hz}, 1 \mathrm{H}), 8.12$ (d, $J=7.5$ $\mathrm{Hz}, 1 \mathrm{H}), 8.09(\mathrm{~m}, 1 \mathrm{H}), 7.73(\mathrm{t}, J=8.1 \mathrm{~Hz}, 1 \mathrm{H}), 4.51$ (s, $2 \mathrm{H}), 4.29(\mathrm{~m}, 1 \mathrm{H}), 2.40(\mathrm{~m}, 2 \mathrm{H}), 2.00(\mathrm{~s}, 3 \mathrm{H}), 1.96(\mathrm{~m}$, 1H), $1.84(\mathrm{~m}, 1 \mathrm{H}), 1.22(\mathrm{~s}, 6 \mathrm{H}), 1.13(\mathrm{~s}, 6 \mathrm{H}) .{ }^{13} \mathrm{C} \mathrm{NMR}(75$ MHz, DMSO- $\left.d_{6}\right): \delta / \mathrm{ppm}=173.35,167.46,162.49,147.98$, 134.73, 132.81, 130.46, 125.22, 123.11, 79.65, 75.69, 72.93, 68.33, 51.31, 31.16, 30.03, 23.90, 14.96. Anal. Calcd for $\mathrm{C}_{20} \mathrm{H}_{28} \mathrm{~N}_{4} \mathrm{O}_{6} \mathrm{~S}: \mathrm{C}, 53.08 ; \mathrm{H}, 6.24 ; \mathrm{N}, 12.38$. Found: C, 53.30; $\mathrm{H}, 6.39$; N, 12.62 .

(2S)-5-guanidino-2-(2-(4,4,5,5-tetramethyl-2-(3nitrophenyl)-4,5-dihydroimidazol- I-yloxy)acetamido) pentanoic acid (6n)

Yield: $300 \mathrm{mg}$ (64\%). $\mathrm{Mp} 68^{\circ} \mathrm{C}-75^{\circ} \mathrm{C}$; ESI/MS (m/z) 478

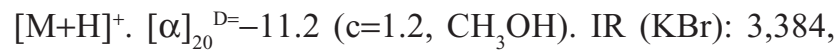
3,450, 2,983, 1,671, 1,535, 1,354, 1,267, 1,173, 1,037, 641, $517 \mathrm{~cm}^{-1}$; ${ }^{1} \mathrm{H}$ NMR (300 MHz, DMSO- $d_{6}$ ): $\delta / \mathrm{ppm}=8.43$ (s, $1 \mathrm{H}), 8.32(\mathrm{~d}, J=6.9 \mathrm{~Hz}, 1 \mathrm{H}), 8.11(\mathrm{~d}, J=6.6 \mathrm{~Hz}, 1 \mathrm{H}), 7.97$ (d, $J=5.7 \mathrm{~Hz}, 1 \mathrm{H}), 7.73(\mathrm{~m}, 2 \mathrm{H}), 7.11(\mathrm{~m}, 4 \mathrm{H}), 4.51(\mathrm{~s}, 2 \mathrm{H})$, $4.18(\mathrm{~m}, 1 \mathrm{H}), 3.07(\mathrm{~m}, 2 \mathrm{H}), 1.73(\mathrm{~m}, 1 \mathrm{H}), 1.58(\mathrm{~m}, 1 \mathrm{H})$, $1.43(\mathrm{~m}, 2 \mathrm{H}), 1.23(\mathrm{~s}, 6 \mathrm{H}) 1.13(\mathrm{~s}, 6 \mathrm{H}) .{ }^{13} \mathrm{C} \mathrm{NMR}(75 \mathrm{MHz}$, DMSO- $\left.d_{6}\right): \delta / \mathrm{ppm}=173.70,167.27,162.46,157.21,148.03$, $134.69,132.83,130.46,125.20,123.09,75.75,72.95,68.36$, 51.97, 49.05, 28.94, 25.52, 23.90, 19.84. Anal. Calcd for $\mathrm{C}_{21} \mathrm{H}_{31} \mathrm{~N}_{7} \mathrm{O}_{6}: \mathrm{C}, 52.82 ; \mathrm{H}, 6.54 ; \mathrm{N}, 20.53$. Found: C, 52.61; $\mathrm{H}, 6.37 ; \mathrm{N}, 20.30$.

(2S)-I -(2-(4,4,5,5-tetramethyl-2-(3-nitrophenyl)-4,5dihydroimidazol- I-yloxy)acetyl)pyrrolidine-2-carboxylic acid (6o)

Yield: $220 \mathrm{mg}(23 \%)$. Mp $112^{\circ} \mathrm{C}-115^{\circ} \mathrm{C}$; ESI/MS (m/z) 417 $[\mathrm{M}-\mathrm{H}]^{-} .[\alpha]_{20}^{\mathrm{D}=}=-64.9\left(\mathrm{c}=1.2, \mathrm{CH}_{3} \mathrm{OH}\right) . \mathrm{IR}(\mathrm{KBr}): 3,420,3,087$, $2,978,2,850,2,780,1,727,1,656,1,532,1,445,1,349,1,268$, $1,162,1,082,822,718 \mathrm{~cm}^{-1} ;{ }^{1} \mathrm{H}$ NMR (300 MHz, DMSO- $\left.d_{6}\right)$ : $\delta / \mathrm{ppm}=8.43(\mathrm{~s}, 1 \mathrm{H}), 8.33(\mathrm{~m}, 1 \mathrm{H}), 8.12(\mathrm{~d}, J=7.2 \mathrm{~Hz}$, $1 \mathrm{H}), 7.73(\mathrm{t}, J=8.1 \mathrm{~Hz}, 2 \mathrm{H}), 4.70(\mathrm{~m}, 2 \mathrm{H}), 4.21(\mathrm{~m}, 1 \mathrm{H})$, $3.45(\mathrm{~m}, 2 \mathrm{H}), 2.07(\mathrm{~m}, 2 \mathrm{H}), 1.86(\mathrm{~m}, 2 \mathrm{H}), 1.24(\mathrm{~s}, 6 \mathrm{H}), 1.12$ (s, 6H). ${ }^{13} \mathrm{C}$ NMR (75 MHz, DMSO- $\left.d_{6}\right): \delta / \mathrm{ppm}=166.23$, 165.87, 162.51, 147.98, 134.72, 132.84, 130.48, 125.18, 123.23, 75.42, 73.06, 68.27, 65.39, 46.49, 45.95, 29.03, 24.77, 23.93, 15.63. Anal. Calcd for $\mathrm{C}_{20} \mathrm{H}_{26} \mathrm{~N}_{4} \mathrm{O}_{6}: \mathrm{C}, 57.41$; H, 6.26; N, 13.39. Found: C, 57.20; H, 6.11; N, 13.16 .

\section{Pain threshold in vivo assay}

Male ICR mice weighing $25 \pm 2 \mathrm{~g}$ were housed in a $12 / 12$ light/dark cycle at a room temperature of $21^{\circ} \mathrm{C} \pm 2^{\circ} \mathrm{C}$ for 
2 days before being used. Food and tap water were supplied ad libitum. The ethical guidelines described in the National Institutes of Health Guide for the Care and Use of Laboratory Animals were followed throughout the experiments.

The mice were randomly divided into three groups of 12 mice, named the test group, vehicle control group, and positive control group. The mice in the vehicle control group were orally given a single dose of normal saline (NS), in the positive group they were orally given a single dose of $167 \mu \mathrm{mol} / \mathrm{kg}$ of aspirin, while in the test group they were orally given a single dose of $10 \mu \mathrm{mol} / \mathrm{kg}, 3.3 \mu \mathrm{mol} / \mathrm{kg}$, or $1 \mu \mathrm{mol} / \mathrm{kg}$ of $6 \mathrm{a}-\mathrm{o}$ in NS. The analgesic effects of $6 \mathrm{a}-\mathrm{o}$ were evaluated by the tail-flick test. The basic pain threshold value of each mouse was measured four times.

Thirty minutes after administration, the pain thresholds were measured at 30-minute intervals. This measurement was carried out for 180 minutes. The potency of analgesia was indicated by pain threshold variation. The values were calculated according to the equation $\mathrm{PTV}=\mathrm{AAPT} / \mathrm{BPT}$, where $\mathrm{PTV}=$ pain threshold variation, $\mathrm{BPT}=$ basic pain threshold, and AAPT = pain threshold after administration - basic pain threshold. All values of the pain threshold variation for each animal were averaged and constituted one sample.

\section{Anti-inflammatory assay in vivo}

Male ICR mice weighing $25 \pm 2 \mathrm{~g}$ were housed in a $12 / 12$ light/dark cycle at a room temperature of $21^{\circ} \mathrm{C} \pm 2^{\circ} \mathrm{C}$ for 2 days before being used. Food and tap water were supplied ad libitum. The ethical guidelines described in the National Institutes of Health Guide for the Care and Use of Laboratory Animals were followed throughout the experiments.

The mice were randomly divided into three groups of 12 mice, named the test group, vehicle control group, and positive control group. Each of the mice in the vehicle control group, positive control group, and test group was orally given a single dose of NS, $167 \mu \mathrm{mol} / \mathrm{kg}$ of aspirin in NS, and $10 \mu \mathrm{mol} / \mathrm{kg}, 3.3 \mu \mathrm{mol} / \mathrm{kg}$, or $1 \mu \mathrm{mol} / \mathrm{kg}$ of $6 \mathrm{a}-\mathrm{s}$ in $\mathrm{NS}$, respectively. Thirty minutes later, $0.03 \mathrm{~mL}$ of xylene was applied to both the anterior and posterior surfaces of the right ear. The left ear was considered as a control. Two hours after xylene application, mice were sacrificed and both ears were removed. Using a cork borer with a diameter of $7 \mathrm{~mm}$, several circular sections were taken and weighed. The increase in weight caused by the irritant was measured through subtracting the weight of the untreated left ear section from that of the treated right ear section. The statistical analysis of the data was carried out by use of ANOVA test. $P<0.05$ was considered significant.

\section{D-QSAR}

The 3D-QSAR of 15 anti-inflammatory agents was analyzed with Discovery Studio 4.0. A subset of 12 anti-inflammatory agents was utilized as a training set for QSAR modeling. To assess the predictive power of the resulting QSAR models, the remaining three antithrombotic agents were employed as a testing set. The Diverse Molecules protocol in Discovery Studio 4.0 was used to build the training and test sets. The inhibition values of the 15 anti-inflammatory agents were represented with \% inhibition, and used for QSAR analyses as the response variable. The CHARMm force field was selected for the agents. The electrostatic potential and the van der Waals potential were treated as separate terms: $a+1 e$ point charge is used as the electrostatic potential probe, and distance-dependent dielectric constant is used to mimic the solvation effect; a carbon atom with a $1.73 \AA$ radius is used as a probe for the van der Waals potential. The truncation for both the steric and the electrostatic energies was set to $30 \mathrm{kcal} / \mathrm{mol}$. The standard parameters implemented in Discovery Studio 4.0 were used. A partial least-squares model was built using energy grids as descriptors. QSAR models were built using the create 3D-QSAR protocol of Discovery Studio 4.0. The inhibition values for the 15 agents are $6 a=25.56 \%, 6 b=17.07 \%, 6 c=35.95 \%, 6 d=20.55 \%$, $6 \mathrm{e}=20.75 \%, 6 \mathrm{f}=63.98 \%, 6 \mathrm{~g}=39.37 \%, 6 \mathrm{~h}=35.45 \%, 6 \mathrm{i}=$ $11.35 \%, 6 \mathrm{j}=20.92 \%, 6 \mathrm{k}=-4.10 \%, 6 \mathrm{l}=42.93 \%, 6 \mathrm{~m}=32.60 \%$, $6 \mathrm{n}=36.57 \%, 6 \mathrm{o}=28.87 \%$.

\section{Molecular docking}

In the molecular docking, the structure of COX-2 enzyme was treated as rigid and prepared by AutoDockTools 1.5L; ie, merging nonpolar hydrogens and assigning gasteiger charges and autodock elements. Then, the energy-minimized 3D conformations of 15 anti-inflammatory agents were treated as flexible and prepared by AutoDockTools 1.5; ie, merging nonpolar hydrogens, assigning gasteiger charges, finding root and aromatic carbons, detecting rotatable bonds, and setting torsions. The grid box dimensions were set to $22.5 \AA \times 30$ $\AA \times 30 \AA$ using a grid spacing of $0.375 \AA$ for two average structures.

To determine the probable binding conformations, AutoDock4, a very popular docking program with a high success rate, and the energy-minimized 3D conformations of 15 anti-inflammatory agents were used to dock them into the active site of COX-2 enzyme.

Lamarckian genetic algorithm was used to find the appropriate binding positions, orientations, and conformations of 15 anti-inflammatory agents in the binding site of 
the active site of COX-2 enzyme. The global optimization was started with parameters of a population of 100 randomly positioned individuals. The maximum number of energy evaluations was increased to $2.5 \times 10^{7}$ and the maximum number of generations in the Lamarckian genetic algorithm was increased to $2.7 \times 10^{5}$. A Solis and Wets local search was performed with a maximum number of 3,000. During each simulation experiment, 256 runs were carried out for each antithrombotic agent. The resulting 256 conformations of 15 anti-inflammatory agents were scored by the lowest binding energy and clustered using a root mean squared tolerance of $2.0 \AA$.

\section{Results and discussion Preparation of TMNPIZA}

1 - (4,4,5,5-tetramethyl-2 - (3 - nitrophenyl) - 4, 5dihydroimidazol-1-yl)oxyacetyl-L-amino acids (TMNPIZA, $6 \mathrm{a}-\mathrm{o})$ were synthesized with the synthetic route of Figure 2. In brief, after the bromination of 2-nitropropane, the formed 2,3-dimethyl-2,3-dinitrobutane (73\% yield) was reduced to 2,3-dihydroxylamino-2,3-dimethylbutane in 63\% yield, which was condensed with 3-nitrobenzaldehyde to provide 1,3-dihydro-xyl-2-(3-nitrophen-1-yl)-4,4,5,5-tetramethylimidazolidine ([1], 75\% yield). The dehydrolysis of
(1) formed 1-hydroxyl-2-(3-nitrophen-1-yl)-4,4,5,5tetramethylimida-zoline (2) in $66 \%$ yield. The alkylation of (2) with $\mathrm{BrCH}_{2} \mathrm{CO}_{2} \mathrm{C}_{2} \mathrm{H}_{5}$ provided ethyl 2-(4,4,5,5-tetramethyl2-(3-nitrophenyl)-4,5-dihydroimidazol-1-yl)-1-oxyacetate (3) in $82 \%$ yield. The saponification of (3) formed (4) $(87 \%$ yield), which was coupled with L-amino acid benzylesters to form 1-(4,4,5,5-tetramethyl-2-(3-nitro-phenyl)-4,5-dihydroimidazol-1-yloxy)acetyl-L-amino acid benzylesters ( $5 \mathrm{a}-\mathrm{o})$ in $42 \%-90 \%$ yields. The saponification of $5 \mathrm{a}-\mathrm{o}$ provided TMNPIZA (6a-o) in 20\%-87\% yields. The common reaction condition and the acceptable yield suggest that this synthetic route is suitable for preparing TMNPIZA.

\section{Analgesic activities of TMNPIZA}

The in vivo oral analgesic evaluation was performed on a tail-flick mouse model for a single dose of $10 \mu \mathrm{mol} / \mathrm{kg}$ of TMNPIZA, a single dose of $167 \mu \mathrm{mol} / \mathrm{kg}$ of aspirin (positive control), or a single dose of $10 \mathrm{~mL} / \mathrm{kg}$ of NS (negative control). The values of basic pain threshold (BPT) and the pain threshold after administration (PTAD) of the mice were tested at 30-minutes intervals for 180 minutes. Using BPT, PTAD, and AAPT (PTAD minus BPT) values, the pain threshold variation $(\mathrm{PTV})$ was calculated based on $\mathrm{PTV}=\mathrm{AAPT} / \mathrm{BPT}$. The data are listed in Figure 3.

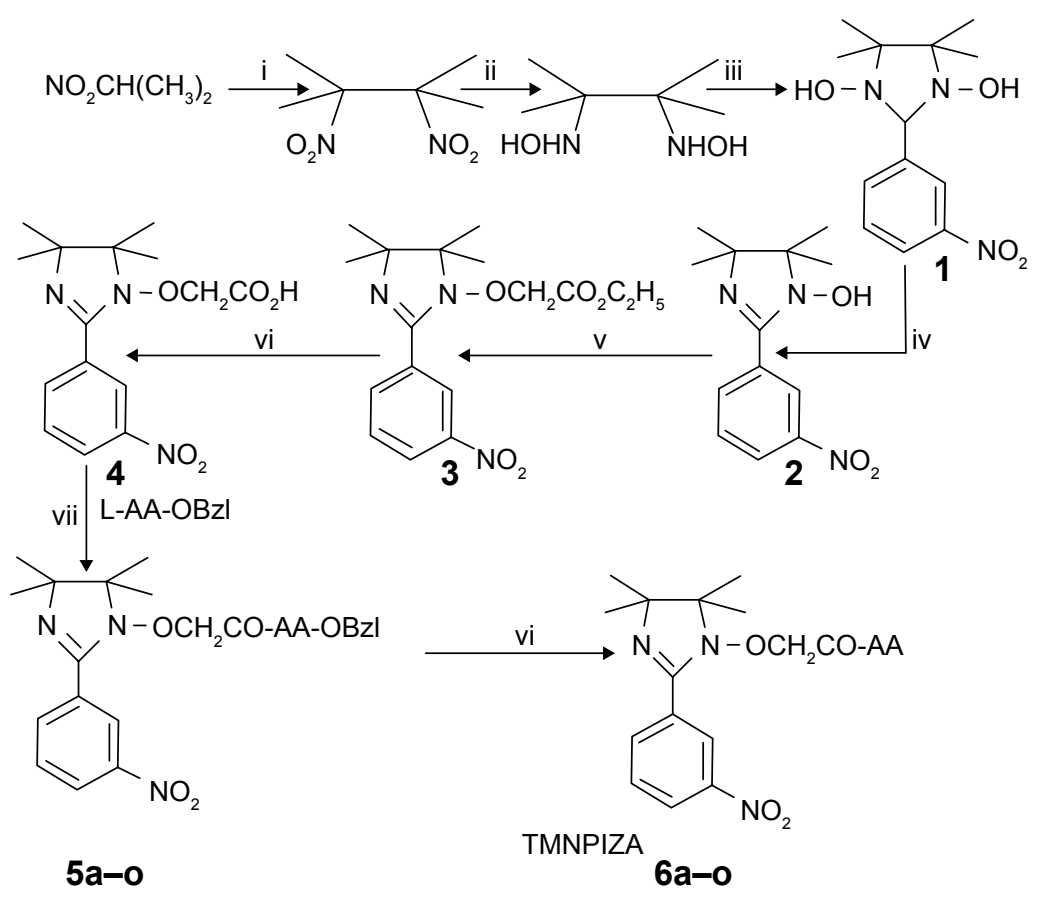

Figure 2 Synthetic route of TMNPIZA.

Notes: Reagents used in the reactions: $\mathrm{Br} 2, \mathrm{NaOH}$, ethanol, $\mathrm{Zn}, \mathrm{NH} 4 \mathrm{Cl}, \mathrm{THF}, \mathrm{OHC}-\mathrm{C} 6 \mathrm{H} 4-\mathrm{NO} 2-\mathrm{m}, \mathrm{methanol}, \mathrm{MgSO} 4, \mathrm{NaH}, \mathrm{BrCH} 2 \mathrm{CO} 2 \mathrm{C} 2 \mathrm{H} 5, \mathrm{NaOH}$ in methanol (4 M), DCC, HOBt, and NMM. (i) $\mathrm{Br}_{2}, \mathrm{NaOH}$, ethanol; ii) $\mathrm{Zn}, \mathrm{NH}_{4} \mathrm{Cl}$, THF; iii) $\mathrm{OHC}-\mathrm{C}_{6} \mathrm{H}_{4}-\mathrm{NO}_{2}-\mathrm{m}$, methanol; iv) anhydrous $\mathrm{MgSO}$, anhydrous $\mathrm{THF}$; v) $\mathrm{NaH}, \mathrm{BrCH}_{2} \mathrm{CO}_{2} \mathrm{C}_{2} \mathrm{H}_{5}$, anhydrous THF; vi) $\mathrm{NaOH}$ in methanol (4 M); vii) DCC, HOBt, NMM, anhydrous THF. In 5a and 6a AA=L-Ile; 5b and 6b AA=L-Val; 5c and 6c $\mathrm{AA}=\mathrm{L}-\mathrm{Leu} ; 5 \mathrm{~d}$ and $6 \mathrm{~d} \mathrm{AA}=\mathrm{L}-\mathrm{Ala} ; 5 \mathrm{e}$ and $6 \mathrm{e} \mathrm{AA}=\mathrm{Gly} ; 5 \mathrm{f}$ and $6 \mathrm{f} \mathrm{AA}=\mathrm{L}-\mathrm{Tyr} ; 5 \mathrm{~g}$ and $6 \mathrm{~g} \mathrm{AA}=\mathrm{L}-\mathrm{Glu} ; 5 \mathrm{~h}$ and $6 \mathrm{~h} \mathrm{AA}=\mathrm{L}-\mathrm{Lys} ; 5 \mathrm{i}$ and $6 \mathrm{i} \mathrm{AA}=\mathrm{L}-\mathrm{Asn} ; 5 \mathrm{j}$ and $6 \mathrm{j} \mathrm{AA}=\mathrm{L}-\mathrm{Gln}$; $5 \mathrm{k}$ and $6 \mathrm{k}$ AA=L-Thr; $5 \mathrm{l}$ and $6 \mathrm{IAA}=\mathrm{L}-\mathrm{Cys} ; 5 \mathrm{~m}$ and $6 \mathrm{~m} \mathrm{AA}=\mathrm{L}-$ Met; $5 \mathrm{n}$ and $6 \mathrm{n} \mathrm{AA}=\mathrm{L}-\mathrm{Arg}$; 50 and $60 \mathrm{AA}=\mathrm{L}-\mathrm{Pro}$ residues.

Abbreviations: AA, amino acid; TMNPIZA, I-(4,4,5,5-tetramethyl-2-(3-nitrophenyl)-4,5-dihydroimidazol-I-yl)oxyacetyl-L-amino acids; Bzl, benzyl. 


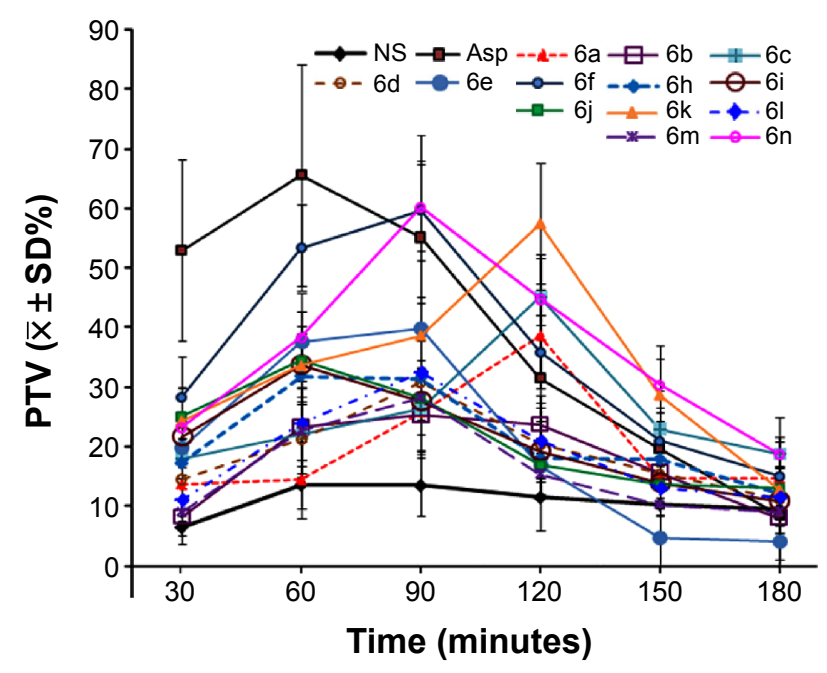

Figure 3 Effect of TMNPIZA (6a-o) on the PTV of mice, $n=12$. The analgesic action of $6 \mathrm{c}, 6 \mathrm{e}, 6 \mathrm{f}$, and $6 \mathrm{n} ; 6 \mathrm{~h}$ and $6 \mathrm{k}$; and $6 \mathrm{a}, 6 \mathrm{~d}, 6 \mathrm{i}, 6 \mathrm{j}$, and $6 \mathrm{l}$ continued for 180 minutes, 150 minutes, and 120 minutes, respectively (compared with NS, $P<0.05$ or $P<0.01$ ), 60 exhibited no analgesic action at the 30 -minute point, $6 \mathrm{~g}$ exhibited no analgesic action at the 30- and 150-minute points (compared with NS, P>0.05), and the analgesic action of $6 \mathrm{~m}$ continued for 90 minutes (compared with NS, $P<0.05$ or $P<0.0$ I).

Abbreviations: SD, standard deviation; TMNPIZA, I-(4,4,5,5-tetra-methyl-2-(3nitrophenyl)-4,5-dihydroimidazol-I-yl)-oxyacetyl-L-amino acids; PTV, pain threshold variation; NS, normal saline;Asp, aspirin.

It was found that the analgesic action of $6 \mathrm{c}, 6 \mathrm{e}, 6 \mathrm{f}$, and $6 \mathrm{n}$; $6 \mathrm{~h}$ and $6 \mathrm{k}$; and $6 \mathrm{a}, 6 \mathrm{~d}, 6 \mathrm{i}, 6 \mathrm{j}$, and 61 continued 180 minutes, 150 minutes, and 120 minutes, respectively (being significantly higher than NS). Of 15 compounds, 60 exhibited no analgesic action at the 30 -minute point (being equal to that of NS), $6 \mathrm{~g}$ exhibited no analgesic action at the 30-minute and 150-minute points (being equal to that of NS), and the analgesic action of $6 \mathrm{~m}$ continued only for 90 minutes (being significantly higher than NS). These data emphasized that TMNPIZA exhibited analgesic action in a rapid and longacting manner. On the other hand, at different time points, the TMNPIZA offered different activities. At the 60-minute and 90-minute points, $6 \mathrm{f}$ possessed the highest activity. This means that the side chain of the amino acid residue significantly affects the analgesic activity, and when the side chain contains the group $-\mathrm{CH}_{2} \mathrm{C}_{6} \mathrm{H}_{4}-\mathrm{OH}-\mathrm{p}$, the analgesic agent will have desirable activity.

\section{Dose-dependent action of $6 \mathrm{f}$ in analgesic action}

To reveal the dose dependence of the analgesic action, $6 \mathrm{f}$ was selected as a representative of TMNPIZA. Its activities were tested at $10,3.3$, and $1 \mu \mathrm{mol} / \mathrm{kg}$ doses, and the data are shown in Figure 4. At a $10 \mu \mathrm{mol} / \mathrm{kg}$ dose, the analgesic action continued for 180 minutes. At a $3.3 \mu \mathrm{mol} / \mathrm{kg}$ dose, the analgesic action was observed at 60-, 90-, and 120-minute points only, while $1 \mu \mathrm{mol} / \mathrm{kg}$ of dose gave no observeable analgesic action. These data demonstrate that at 60-, 90-, and 120-minute points, $6 \mathrm{f}$ increased the pain threshold in a dose-dependent manner.

\section{Anti-inflammatory activities of TMNPIZA}

To explore the utility of TMNPIZA in treating inflammatory pain, $6 \mathrm{a}-\mathrm{O}$ were assayed on xylene-induced ear edema of mice in vivo. The data are shown in Figure 5. It was

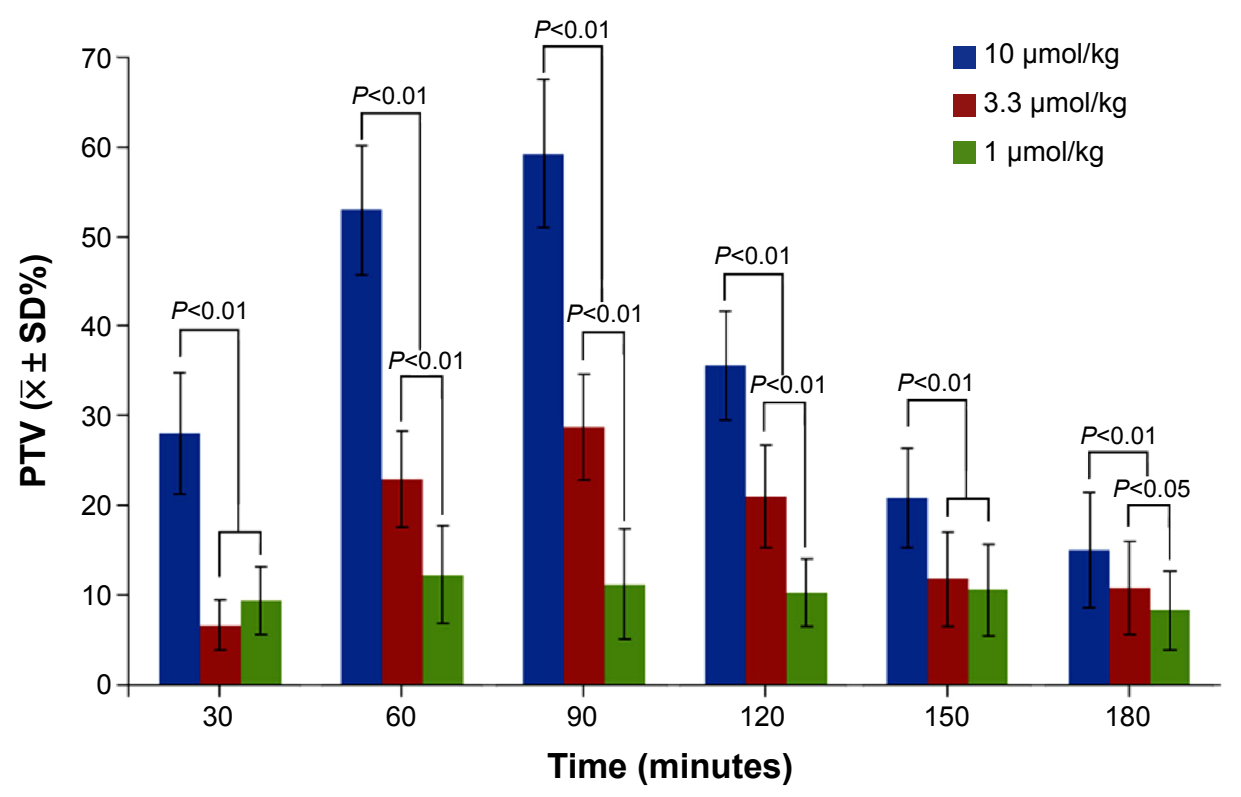

Figure 4 Effect of $6 \mathrm{f}$ dose on the PTV of mice, $\mathrm{n}=12$.

Abbreviations: SD, standard deviation; PTV, pain threshold variation. 


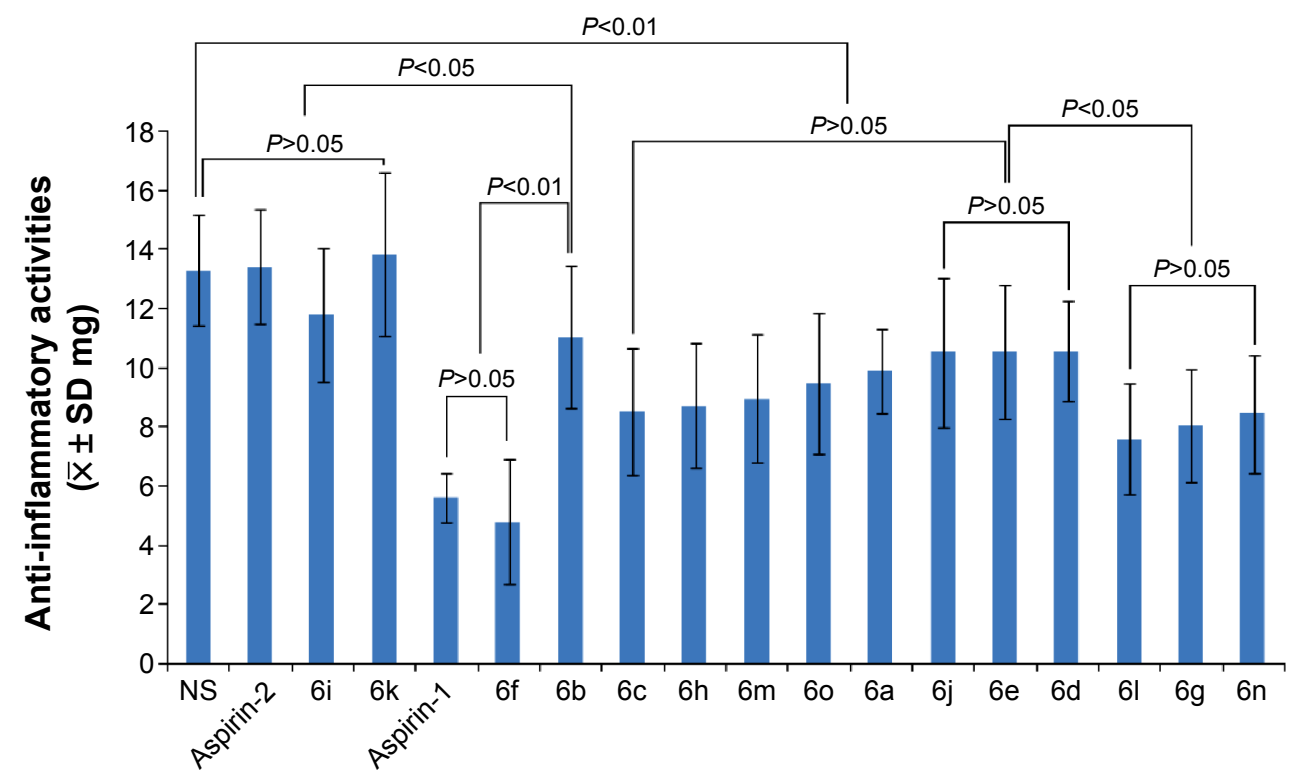

Figure 5 Anti-inflammatory activities of $6 \mathrm{a}-\mathrm{O}$ on xylene-induced ear edema model, $\mathrm{n}=12$; NS=vehicle; doses of aspirin-I and $-2 \mathrm{were} 167 \mu \mathrm{mol} / \mathrm{kg}$ and $16.7 \mu \mathrm{mol} / \mathrm{kg}$, respectively; dose of $6 \mathrm{a}-0$ was $10 \mu \mathrm{mol} / \mathrm{kg}$.

Abbreviations: SD, standard deviation; NS, normal saline.

noted that at $10 \mu \mathrm{mol} / \mathrm{kg}$ of dose, except for $6 \mathrm{i}$ and $6 \mathrm{k}$, all the others exhibited anti-inflammatory action. The activities of $6 \mathrm{~b}(P<0.05)$ and $6 \mathrm{a}, 6 \mathrm{c}-\mathrm{h}, 6 \mathrm{j}$, and $6 \mathrm{l}-\mathrm{o}(P<0.01)$ were significantly higher than those of NS. The activities of $6 \mathrm{~g}, 61$, and $6 \mathrm{n}$ were significantly higher than those of $6 \mathrm{~d}$, $6 \mathrm{e}$, and $6 \mathrm{j}(P<0.05)$. Besides, the activity of $10 \mu \mathrm{mol} / \mathrm{kg}$ (6f) was equal to that of $167 \mu \mathrm{mol} / \mathrm{kg}$ aspirin, suggesting that the effective dose of $6 \mathrm{f}$ was 16.7 -fold lower than that of aspirin.

Of 15 anti-inflammatory agents, $6 \mathrm{f}$ possessed the highest activity. This means that the side chain of the amino acid residue significantly affects the activity, and when the side chain contains the group $-\mathrm{CH}_{2} \mathrm{C}_{6} \mathrm{H}_{4}-\mathrm{OH}-\mathrm{p}$, the anti-inflammatory agent will have desirable activity.

\section{Dose-dependent action of $6 \mathrm{f}$ in anti- inflammatory action}

To reveal the dose dependence of the anti-inflammatory action, $6 \mathrm{f}$ was selected as a representative of TMNPIZA. Its activities were tested at $10,3.3$, and $1 \mu \mathrm{mol} / \mathrm{kg}$ doses, and the data are shown in Figure 6. On a xylene-induced ear edema model, the ear edema of mice in $10 \mu \mathrm{mol} / \mathrm{kg}$ group was significantly lower than that of mice in the $3.3 \mu \mathrm{mol} / \mathrm{kg}$ group $(P<0.01)$, and the ear edema of mice in the $1 \mu \mathrm{mol} / \mathrm{kg}$ group was significantly higher than that of mice in the $3.3 \mu \mathrm{mol} / \mathrm{kg}$ group $(P<0.01)$ and was significantly lower than that of mice in the NS group $(P<0.01)$. Thus, on the xylene-induced ear edema model, $6 \mathrm{f}$ gave a dose-dependent anti-inflammatory response, and its effective dose was $1 \mu \mathrm{mol} / \mathrm{kg}$.

\section{D-QSAR of TMNPIZA inhibiting inflammation}

To rationally design the inhibitors of inflammatory pain afterwards, the 3D-QSAR of the 15 anti-inflammatory agents was analyzed, for which a training set of 12 agents $(6 \mathrm{~b}-\mathrm{g}$, $6 \mathrm{i}$, and $6 \mathrm{k}-\mathrm{o})$ and a test set of three agents $(6 \mathrm{a}, 6 \mathrm{~h}$, and $6 \mathrm{j}$ ) was set. Their $\%$ inhibition values (from $-4.10 \%$ to $63.98 \%$ )

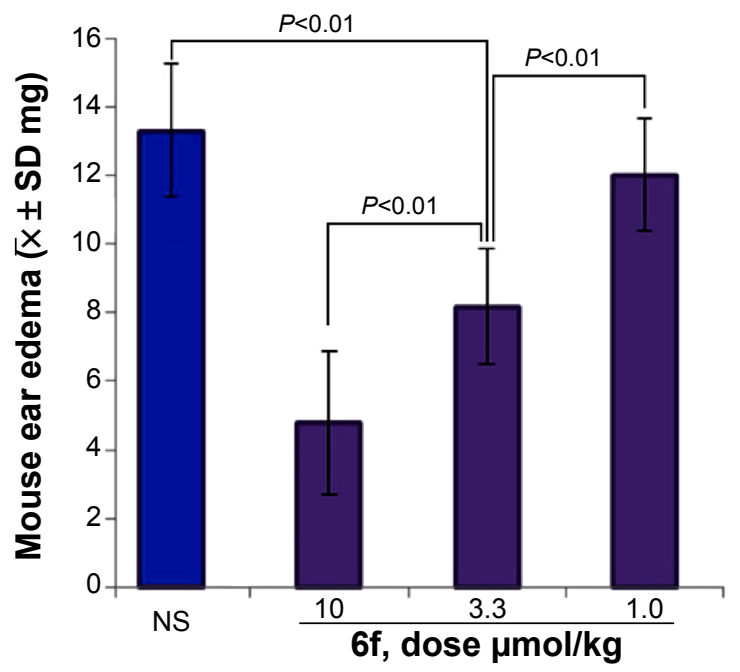

Figure 6 Effect of various doses of $6 f$ on xylene-induced mouse ear edema, $n=12$. Abbreviations: SD, standard deviation; NS, normal saline. 


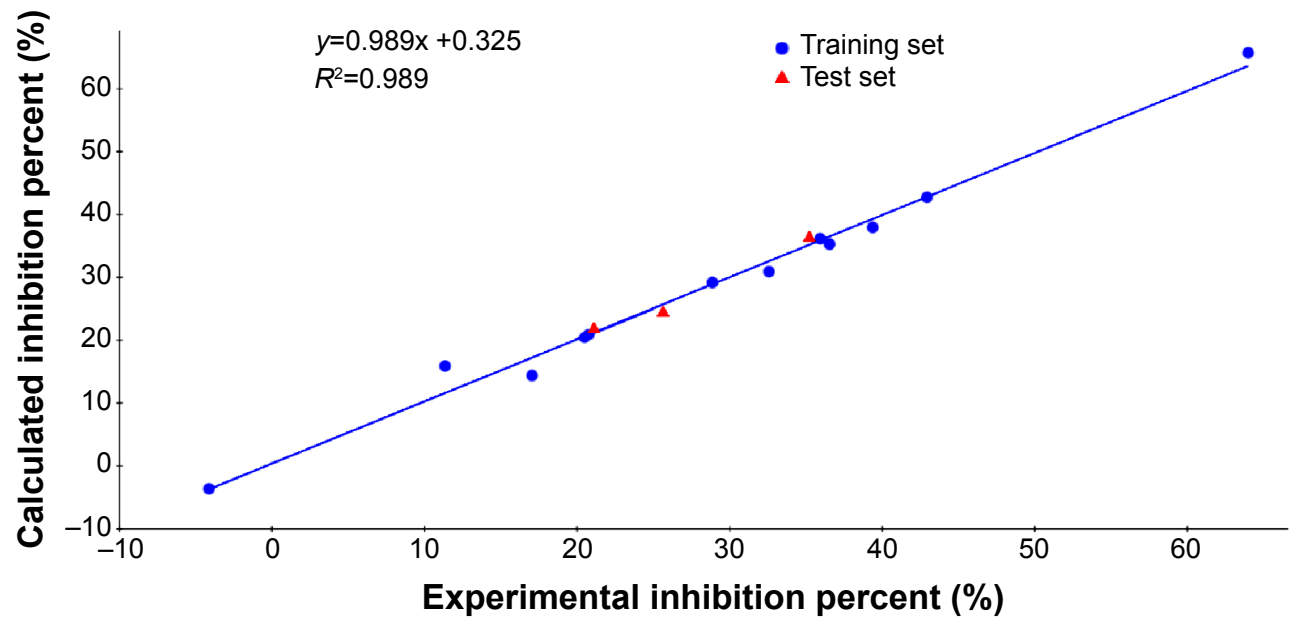

Figure 7 Graphical relationship of experimental and predicted inhibitions of 12 agents in the training set and three agents in the test set.

in inhibiting xylene-induced ear edema of mice were used, and the 3D-QSAR was analyzed according to a standard method. In the analysis, the comparative molecular field analysis (CoMFA) field descriptors were used as independent variables, and the $\%$ inhibition values were used as the dependent variables. The values of $\mathrm{n}, r$, and $r^{2}$ were 12, 0.994 , and 0.989 , respectively. The relationship of the experimental and predicted inhibitions is shown in Figure 7.

With the relationship of Figure 7, the inhibitions of the test set ( $6 \mathrm{a}, 6 \mathrm{~h}$, and $6 \mathrm{j}$ ) were calculated and the relationship of their experimental inhibitions of 6a-, 6h-, and 6j-treated mice $(25.56 \%, 35.45 \%$, and $20.92 \%)$ and the calculated inhibitions of $6 \mathrm{a}-, 6 \mathrm{~h}$-, and $6 \mathrm{j}$-treated mice $(24.66 \%, 35.63 \%$, and $22.86 \%)$ are also shown in Figure 7. The prediction errors were $-3.5 \%$,
$0.5 \%$, and $9.3 \%$, respectively, suggesting the the relationship of Figure 7 had good validity. The visual 3D-QSAR contains steric fields, the green region mirroring a bulky group increases the activity and the yellow region mirroring bulky group decreases the activity (Figure 8A), and electrostatic fields, the blue region mirroring electron-donating group increases the activity and the red region mirroring an electronwithdrawing group decreases the activity (Figure 8B).

According to Figure 8B, all side chains of the amino acid residues of TMNPIZA is located in the blue region, and the electron-donating side chains will enhance the anti-inflammatory activity.

As mentioned, of 15 agents, $6 \mathrm{f}$ and $6 \mathrm{n}$ had the highest analgesic activity at 60 - and 90 -minute points, and possessed
A

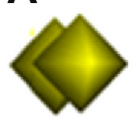

B
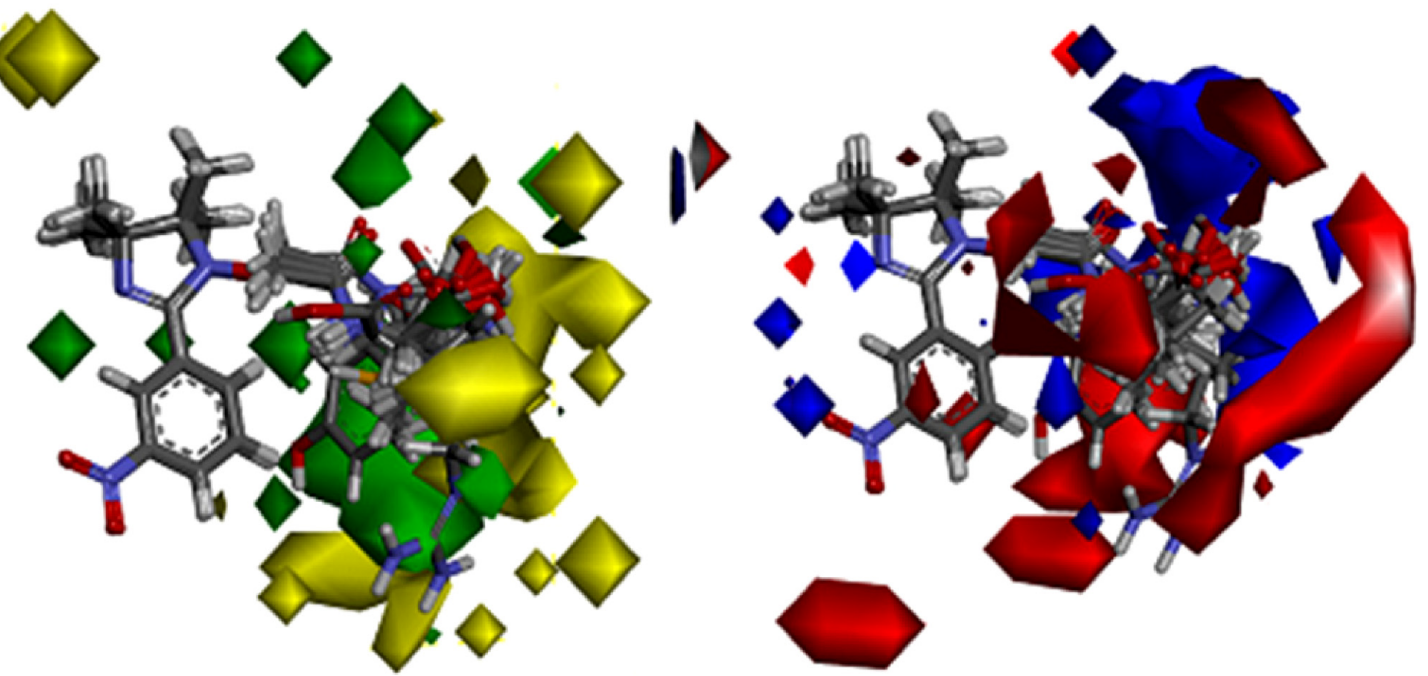

Figure 8 Graphical 3D-QSAR correlating to the steric field (A) and electrostatic field (B) with the chemical environment of I5 anti-inflammatory agents in the active site of COX-2 enzyme.

Abbreviations: 3D-QSAR, three-dimensional quantitative structure-activity relationship; COX-2, cyclooxygenase-2. 
the highest anti-inflammation activity. Based on Figure 8B, it is hypothesized that the p-electron-containing group is the electron-donating group, and when it is located in the blue region, the agent will exhibit good analgesic and antiinflammation activities. Due to the side chain of the amino acid, residue of $6 \mathrm{f}$ contains $-\mathrm{CH}_{2} \mathrm{C}_{6} \mathrm{H}_{4}-\mathrm{OH}-\mathrm{p}$, which is p-electron-containing groups, and is located in the blue region of Figure 8B. 6f not only possesses good analgesic activity but also possesses good anti-inflammatory activity.

\section{Conclusion}

The docking investigation, the in vivo anti-inflammation/ analgesic evaluations, and the 3D-QSAR analysis provided evidence that the 15 novel 1-(4,4,5,5-tetramethyl-2-(3nitrophenyl)-4,5-dihydroimidazol-1-yl)oxyacetyl-L-amino acids should be promising candidates for developing drugs to improve inflammatory pain therapy.

\section{Acknowledgments}

This work was supported by the Beijing Municipal Science and Technology Commission (Z141100002114049), the Project of Construction of Innovative Teams and Teacher Career Development for Universities and Colleges Under Beijing Municipality, the NSFC (81172930, 81270046, 81373265 and 81202412), and Beijing Natural Science Foundation (7132032).

\section{Disclosure}

The authors report no conflicts of interest in this work.

\section{References}

1. Li X, Bantel C, Conklin D, Conklin D, Childers SR, Eisenach JC. Repeated dosing with oral allosteric modulator of adenosine A1 receptor produces tolerance in rats with neuropathic pain. Anesthesiology. 2004; 100(4):956-961.

2. Grim TW, Ghosh S, Hsu KL, Cravatt BF, Kinsey SG, Lichtman AH. Combined inhibition of FAAH and COX produces enhanced antiallodynic effects in mouse neuropathic and inflammatory pain models. Pharmacol Biochem Behav. 2014;124:405-411.
3. Kam YL, Back SK, Kang B, et al. HYP-1, a novel diamide compound, relieves inflammatory and neuropathic pain in rats. Pharmacol Biochem Behav. 2012;103(1):33-42.

4. Paulino N, Rodrigues NC, Pardi PC, et al. Evaluation of anti-inflammatory effect of synthetic 1,5-bis(4-acetoxy-3-methoxyphenyl)-1,4-pentadien3-one, HB2. Bioorg Med Chem. 2009;17(13):4290-4295.

5. Buvanendran A, Reuben SS, Kroin JS. Recent advances in nonopioid analgesics for acute pain management. Tech Reg Anesth Pain Manag. 2007;11(1):19-26.

6. García-Martínez C, Fernández-Carvajal A, Valenzuela B, et al. Design and characterization of a noncompetitive antagonist of the transient receptor potential vanilloid subunit 1 channel with in vivo analgesic and anti-inflammatory activity. Pain. 2006;7(10):735-746.

7. Sobczak M, Pilarczyk A, Jonakowski M, Lipkowski AW, Fichna J. Anti-inflammatory and antinociceptive action of the dimeric dimericenkephalin peptide biphalin in the mouse model of colitis: new potential treatment of abdominal pain associated with inflammatory inflammatory bowel diseases. Peptides. 2014;60:102-106.

8. Cao D, Zhang Z, Xie RG, Jiang BC1, Ji RR, Gao YJ. Chemokine CXCL1 enhances inflammatory pain and increases NMDA receptor activity and COX-2 expression in spinal cord neurons via activation of CXCR2. Exp Neurol. 2014;261:328-336.

9. Kono M, Matsumoto T, Imaeda T, et al. Design, synthesis, and biological evaluation of a series of piperazine ureas as fatty acid amide hydrolase inhibitors. Bioorg Med Chem. 2014;22(4):1468-1478.

10. Da Silva YKC, Augusto CV, de Castro Barbosa ML, et al. Synthesis and pharmacological evaluation of pyrazine $\mathrm{N}$-acylhydrazone derivatives designed as novel analgesic and anti-inflammatory drug candidates. Bioorg Med Chem. 2010;18(14):5007-5015.

11. Palkar MB, Singhai AS, Ronad PM, et al. Synthesis, pharmacological screening and in silico studies of new class of diclofenac analogues as a promising anti-inflammatory agents. Bioorg Med Chem. 2014;22(10):2855-2866.

12. Khalil NA, Ahmed EM, Mohamed KO, Nissan YM, Zaitone SA. Synthesis and biological evaluation of new pyrazolone-pyridazine conjugates as anti-inflammatory and analgesic agents. Bioorg Med Chem. 2014;22(7):2080-2089.

13. Irannejad H, Kebriaieezadeh A, Zarghi A, et al. Synthesis, docking simulation, biological evaluations and 3D-QSAR study of 5-Aryl-6(4-methylsulfonyl)-3-(metylthio)-1,2,4-triazine as selective cyclooxygenase-2 inhibitors. Bioorg Med Chem. 2014;22(2):865-873.

14. Zhao M, Li Z, Peng L, et al. A new class of analgesic agents toward prostacyclin receptor inhibition: Synthesis, biological studies and QSAR analysis of 1-hydroxyl-2-substituted phenyl-4,4,5,5-tetramethylimidazolines. Eur J Med Chem. 2008;43(5):1048-1058.

15. Zhao M, Li Z, Peng L, Tang YR, et al. Novel 1-oxyl-2-substitutedphenyl4,4,5,5-tetramethylimidazolines: synthesis, selectively analgesic action, and QSAR analysis. Bioorg Med Chem. 2007;15(8):2815-2826.
Drug Design, Development and Therapy

\section{Publish your work in this journal}

Drug Design, Development and Therapy is an international, peerreviewed open-access journal that spans the spectrum of drug design and development through to clinical applications. Clinical outcomes, patient safety, and programs for the development and effective, safe, and sustained use of medicines are a feature of the journal, which

\section{Dovepress}

has also been accepted for indexing on PubMed Central. The manuscript management system is completely online and includes a very quick and fair peer-review system, which is all easy to use. Visit http://www.dovepress.com/testimonials.php to read real quotes from published authors. 